\title{
A Note on the Life-span of Classical Solutions to Nonlinear Wave Equations in Four Space Dimensions
}

\section{Li TA-TSIEN (LI DA-QIAN) \& ZHOU YI}

\begin{abstract}
In this paper we prove that in the four space dimensional case, L. Hörmander's estimate $\tilde{T}(\varepsilon) \geq \exp \left\{A \varepsilon^{-1}\right\}$ ( $A>0$, constant) can be improved by $\tilde{T}(\varepsilon) \geq \exp \left\{A \varepsilon^{-2}\right\}$ on the lower bound of the life-span $\tilde{T}(\varepsilon)$ of classical solutions to the Cauchy problem with small initial data $\left(u, u_{t}\right)(0, x)=$ $\varepsilon(\varphi(x), \psi(x))$ for nonlinear wave equations of the form $\square u=$ $F\left(u, D u, D_{x} D u\right)$, where $F(\hat{\lambda})=O\left(|\hat{\lambda}|^{2}\right)$ in a neighbourhood of $\hat{\lambda}=0$.
\end{abstract}

1. Introduction. Consider the Cauchy problem for fully nonlinear wave equations

$$
\begin{aligned}
& \square u=F\left(u, D u, D_{x} D u\right), \\
& t=0: u=\varepsilon \varphi(x), u_{t}=\varepsilon \psi(x),
\end{aligned}
$$

where

$$
\square=\frac{\partial^{2}}{\partial t^{2}}-\sum_{i=1}^{n} \frac{\partial^{2}}{\partial x_{i}^{2}}
$$

is the wave operator,

$$
D_{x}=\left(\frac{\partial}{\partial x_{1}}, \ldots, \frac{\partial}{\partial x_{n}}\right), \quad D=\left(\frac{\partial}{\partial t}, \frac{\partial}{\partial x_{1}}, \ldots, \frac{\partial}{\partial x_{n}}\right),
$$

$\varphi, \psi \in C_{0}^{\infty}\left(\mathbb{R}^{n}\right)$ and $\varepsilon>0$ is a small parameter.

Let

$$
\hat{\lambda}=\left(\lambda ;\left(\lambda_{i}\right), i=0,1, \ldots, n ;\left(\lambda_{i j}\right), i, j=0,1, \ldots, n, i+j \geq 1\right) .
$$


Suppose that in a neighbourhood of $\hat{\lambda}=0$, say, for $|\hat{\lambda}| \leq 1$, the nonlinear term $F=F(\hat{\lambda})$ in (1.1) is a sufficiently smooth function satisfying

$$
F(\hat{\lambda})=O\left(|\hat{\lambda}|^{1+\alpha}\right)
$$

where $\alpha$ is an integer $\geq 1$.

Our aim is to study the life-span of classical solutions to (1.1)-(1.2). By definition, the life-span $\tilde{T}(\varepsilon)=\sup \tau$ for all $\tau>0$ such that there exists a classical solution to (1.1)-(1.2) on $0 \leq t \leq \tau$.

In the general case that the nonlinear term $F$ may explicitly depend on $u: F=F\left(u, D u, D_{x} D u\right)$, for all integers $n, \alpha$ with $n \geq 1$ and $\alpha \geq 1$, the lower bound of the life-span of classical solutions to (1.1)-(1.2) was studied by D. Christodoulou [1], Li Ta-tsien and Chen Yun-mei [2], L. Hörmander [3], H. Lindblad [4], Li Ta-tsien and Yu Xin [5], Li Ta-tsien and Zhou Yi [6], Li Tatsien, Yu Xin and Zhou Yi [7]-[9], Li Ta-tsien and Zhou Yi [10]-[12], etc., and a summary of all the results obtained can be found in Li Ta-tsien and Chen Yu-mei [13] and Li Ta-tsien [14]. All these lower bounds, except in the case $n=4, \alpha=1$ and $\partial_{u}^{2} F(0,0,0) \neq 0$, have been known to be sharp, cf. H. Lindblad [15], Zhou Yi [16]-[18], etc.

In the case $n=4, \alpha=1$ and $\partial_{u}^{2} F(0,0,0) \neq 0$, the following lower bound of the life-span

$$
\tilde{T}(\varepsilon) \geq \exp \left\{A \varepsilon^{-1}\right\}
$$

where $A$ is a positive constant independent of $\varepsilon$, was obtained first by L. Hörmander [3] and then, by means of the global iteration method, by $\mathrm{Li}$ Ta-tsien and Yu Xin [5]. In this paper we shall prove that (1.7) can be improved by

$$
\tilde{T}(\varepsilon) \geq \exp \left\{A \varepsilon^{-2}\right\},
$$

where $A$ is a positive constant independent of $\varepsilon$.

In order to illustrate estimate (1.8), as the 'worst' case of equation (1.1), we consider the equation

$$
\square u=u^{2}
$$

which can be regarded as a special case $(p=2)$ of the following equation

$$
\square u=|u|^{p}
$$

$$
(p>1) .
$$

When $n=3$, F. John [19] proved that if $p>1+\sqrt{2}$, then Cauchy problem (1.10) and (1.2) admits a unique global solution on $t \geq 0$, provided that $\varepsilon>0$ 
is suitably small; while, if $1<p<1+\sqrt{2}$, then any nontrivial solution with compact support to equation (1.10) must blow up in a finite time. Thus, in the case $n=3$, the critical value of $p$ is equal to $p_{0}(3)=1+\sqrt{2}$. In general, as suggested and studied by R. T. Glassey [20]-[21], T. C. Sideris [22], J. Schaeffer [23] etc., in $n$ space dimensions, $p_{0}(n)$, the critical value of $p$, should be the positive root of the following quadratic equation

$$
(n-1) p^{2}-(n+1) p-2=0 .
$$

In particular, we have $p_{0}(4)=2$. Hence, equation (1.9) corresponds to the critical value of $p$ in four space dimensions.

When $p=p_{0}(n)$ with $n=2,3$, Zhou Yi [17]-[18] proved that the life-span $\tilde{T}(\varepsilon)$ of solutions to Cauchy problem (1.10) and (1.2) satisfies

$$
\tilde{T}(\varepsilon) \approx \exp \left\{A \varepsilon^{-p(p-1)}\right\},
$$

where $A$ is a positive constant independent of $\varepsilon$; namely, there exist two positive constants $A_{1}$ and $A_{2}$ independent of $\varepsilon$ such that

$$
\exp \left\{A_{1} \varepsilon^{-p(p-1)}\right\} \leq \tilde{T}(\varepsilon) \leq \exp \left\{A_{2} \varepsilon^{-p(p-1)}\right\} .
$$

We guess that (1.12) still holds in the case $n=4$. If so, in four space dimensions the life-span of solutions to Cauchy problem (1.9) and (1.2) should satisfy

$$
\tilde{T}(\varepsilon) \approx \exp \left\{A \varepsilon^{-2}\right\} .
$$

It is just this consideration which leads us to prove (1.8) for Cauchy problem (1.1)-(1.2) and to believe that the lower bound of the life-span is sharp.

We point out that under certain hypotheses on the initial data (1.2), T. C. Sideris [24] recently proved that in four space dimensions the life-span of solutions to Cauchy problem (1.9) and (1.2) possesses the following upper bound

$$
\tilde{T}(\varepsilon) \leq \exp \left\{B \varepsilon^{-4}\right\},
$$

where $B$ is a positive constant independent of $\varepsilon$.

In order to prove the desired conclusion, by differentiation, it suffices to consider the Cauchy problem for the following general kind of quasilinear wave equations:

$$
\begin{aligned}
& \square u=\sum_{i, j=1}^{4} b_{i j}(u, D u) u_{x_{i} x_{j}}+2 \sum_{j=1}^{4} a_{0 j}(u, D u) u_{t x_{j}}+F_{0}(u, D u), \\
& t=0: u=\varepsilon \varphi(x), u_{t}=\varepsilon \psi(x),
\end{aligned}
$$


where $x=\left(x_{1}, x_{2}, x_{3}, x_{4}\right), \square u=\partial^{2} / \partial t^{2}-\sum_{i=1}^{4} \partial^{2} / \partial x_{i}^{2}, \varepsilon>0$ is a small parameter,

$$
\varphi, \psi \in C_{0}^{\infty}\left(\mathbb{R}^{4}\right)
$$

and without loss of generality we may suppose that

$$
\operatorname{supp}\{\varphi, \psi\} \subseteq\{x|| x \mid \leq 1\} .
$$

Moreover, for $|\widetilde{\lambda}| \leq 1$, where $\widetilde{\lambda}=\left(\lambda ;\left(\lambda_{i}\right), i=0,1, \ldots, 4, b_{i j}(\widetilde{\lambda}), a_{0 j}(\widetilde{\lambda})\right.$ and $F_{0}(\widetilde{\lambda})$ are sufficiently smooth functions satisfying

$$
\begin{array}{ll}
b_{i j}(\widetilde{\lambda})=b_{j i}(\widetilde{\lambda}), & i, j=1, \ldots, 4, \\
b_{i j}(\widetilde{\lambda}), a_{0 j}(\widetilde{\lambda})=O(|\widetilde{\lambda}|), & i, j=1, \ldots, 4, \\
F_{0}(\widetilde{\lambda})=O\left(|\widetilde{\lambda}|^{2}\right) &
\end{array}
$$

and

$$
\sum_{i, j=1}^{4} a_{i j}(\widetilde{\lambda}) \xi_{i} \xi_{j} \geq m_{0}|\xi|^{2}, \quad \forall \xi \in \mathbb{R}^{4}
$$

where $m_{0}$ is a positive constant and

$$
a_{i j}(\widetilde{\lambda})=\delta_{i j}+b_{i j}(\widetilde{\lambda}),
$$

where $\delta_{i j}$ is the Kronecker delta.

Let's briefly explain the main technical points of our method here. As mentioned above, L. Hörmander's estimate (1.7) was reproved by Li Ta-tsien and $\mathrm{Yu}$ Xin [5]. The key inequality in [5] will be presented in Theorem 3.4. Based on a Sobolev embedding theorem in the radial direction (Theorem 2.10), the idea of the proof of Theorem 3.4 can be applied to get Theorem 3.5 and Theorem 3.6 which will be very useful in the sequel.

Since the problem under consideration corresponds to the critical case, we need some further refined estimates. For this purpose, noting that the initial data have compact support, a version of Huyghens' principle (Lemma 3.1) will be used to improve some known inequalities. For instance, Hörmander's $L^{1}-L^{\infty}$ estimate (Lemma 3.7) will be improved by Lemma 3.8 and Li-Yu's inequality (Theorem 3.4) by Lemma 3.10 and Theorem 3.11.

In order to illustrate that these improvements do play an important role in the proof, we consider Cauchy problem (1.9) and (1.2) with (1.17). The formal expansion of the solution gives

$$
u=\varepsilon u_{0}+\varepsilon^{2} u_{1}+\cdots,
$$


where $u_{0}$ is the solution to the following linear problem:

$$
\begin{aligned}
& \square u_{0}=0, \\
& t=0: u_{0}=\varphi, u_{0 t}=\psi ;
\end{aligned}
$$

while $u_{1}$ satisfies

$$
\begin{aligned}
& \square u_{1}=u_{0}^{2}, \\
& t=0: u_{1}=0, u_{1 t}=0 .
\end{aligned}
$$

By taking $\ell<\frac{1}{2}$ and $\ell=\frac{1}{2}$, respectively, in Lemma 3.7, we get

$$
\left|u_{1}(t, x)\right| \leq C(1+t+|x|)^{-3 / 2}(1+|t-| x||)^{-\ell}
$$

and

$$
\left|u_{1}(t, x)\right| \leq C(1+t+|x|)^{-3 / 2}(1+|t-| x||)^{-1 / 2} \ln (1+t),
$$

where $C$ is a positive constant. Both (1.28) and (1.29) are not sharp. However, Lemma 3.8 can be used to get the improved estimate

$$
\left|u_{1}(t, x)\right| \leq C(1+t+|x|)^{-3 / 2}(1+|t-| x||)^{-1 / 2} .
$$

Noting that $u_{1}$ has a compact support, it follows from (1.30) that

$$
\left\|u_{1}(t, \cdot)\right\|_{L^{2}\left(\mathbb{R}^{4}\right)} \leq C(\ln (1+t))^{1 / 2} .
$$

If we use Theorem 3.4, however, we can only get

$$
\left\|u_{1}(t, \cdot)\right\|_{L^{2}\left(\mathbb{R}^{4}\right)} \leq C \ln (1+t),
$$

which is not sharp.

Similarly, it follows from (1.30) that

$$
\left\|(1+|t-| x||)^{-1 / 2} \chi_{2} u_{1}(t, \cdot)\right\|_{L^{1,2}\left(\mathbb{R}^{4}\right)} \leq C(1+t)^{3 / 2} \ln (1+t),
$$

where $\chi_{2}$ is defined in Theorem 3.4. (1.33) can also be obtained by means of Lemma 3.10.

Moreover, in order to get the desired conclusion, we have to change the iteration scheme in the global iteration method.

In Section 2 we will give some necessary notations and lemmas. Then, certain estimates on the solution to the Cauchy problem for linear wave equations will be established in Section 3. Finally, the main result and its proof will be given in Section 4. 


\section{Preliminaries.}

Following S. Klainerman [25], introduce a set of partial differential operators

$$
\Gamma=(D, L, \Omega),
$$

where $D$ is defined by (1.4),

$$
L=\left(L_{a}, a=0,1, \ldots, n\right)
$$

and

$$
\Omega=\left(\Omega_{i j}, i, j=1, \ldots, n\right)
$$

with

$$
\begin{aligned}
& L_{0}=t \partial_{t}+x_{1} \partial_{x_{1}}+\cdots+x_{n} \partial_{x_{n}}, \\
& L_{i}=t \partial_{x_{i}}+x_{i} \partial_{t},
\end{aligned}
$$$$
i=1, \ldots, n,
$$

and

$$
\Omega_{i j}=x_{i} \partial_{x_{j}}-x_{j} \partial_{x_{i}}, \quad \quad i, j=1, \ldots, n .
$$

As in Li Ta-tsien and $\mathrm{Yu}$ Xin [5], introduce the Banach space $L^{p, q}\left(\mathbb{R}^{n}\right)$ equipped with the norm

$$
\|f(\cdot)\|_{L^{p, q}\left(\mathbb{R}^{n}\right)}=\left\|f(r \xi) r^{(n-1) / p}\right\|_{L^{p}\left(0, \infty ; L^{q}\left(S^{n-1}\right)\right)},
$$

where $f=f(x), x \in \mathbb{R}^{n}, r=|x|$ and $\xi \in S^{n-1}, S^{n-1}$ being the unit sphere in $\mathbb{R}^{n}, 1 \leq p, q \leq+\infty$. It is easy to see that if $p=q$, then $L^{p, q}\left(\mathbb{R}^{n}\right)$ becomes the usual space $L^{p}\left(\mathbb{R}^{n}\right)$. Moreover, Hölder's inequality still holds for the $L^{p, q}\left(\mathbb{R}^{n}\right)$ norm (see Lemma 2.1 in Li Ta-tsien and Yu Xin [5]).

For any integer $N \geq 0$, define

$$
\|u(t, \cdot)\|_{\Gamma, N, p, q, \chi}=\sum_{|k| \leq N}\left\|\chi(t, \cdot) \Gamma^{k} u(t, \cdot)\right\|_{L^{p, q}\left(\mathbb{R}^{n}\right)},
$$

where $1 \leq p, q \leq+\infty, \chi(t, x)$ is the characteristic function of any given set in $\mathbb{R}_{+} \times \mathbb{R}^{n}, k=\left(k_{1}, \ldots, k_{\sigma}\right)$ are multi-indices, $|k|=k_{1}+\cdots+k_{\sigma}, \sigma$ is the number of the partial differential operators in $\Gamma: \Gamma=\left(\Gamma_{1}, \ldots, \Gamma_{\sigma}\right)$ and $\Gamma^{k}=\Gamma_{1}^{k_{1}} \ldots \Gamma_{\sigma}^{k_{\sigma}}$. In what follows we write

$$
\|u(t, \cdot)\|_{\Gamma, N, p, q, \chi}= \begin{cases}\|u(t, \cdot)\|_{\Gamma, N, p, \chi}, & \text { if } p=q, \\ \|u(t, \cdot)\|_{\Gamma, N, p, q}, & \text { if } \chi \equiv 1 \\ \|u(t, \cdot)\|_{p, q, \chi}, & \text { if } N=0\end{cases}
$$

It is easy to prove the following two lemmas. 
Lemma 2.1. For any multi-index $k=\left(k_{1}, \ldots, k_{\sigma}\right)$, we have

$$
\left[\square, \Gamma^{k}\right]=\sum_{|i| \leq|k|-1} A_{k i} \Gamma^{i} \square
$$

and

$$
\left[D, \Gamma^{k}\right]=\sum_{|i| \leq|k|-1} B_{k i} \Gamma^{i} D=\sum_{|i| \leq|k|-1} \widetilde{B}_{k i} D \Gamma^{i}
$$

where [, ] stands for the Poisson bracket, $i=\left(i_{1}, \ldots, i_{\sigma}\right)$ are multi-indices, $\square$ is the wave operator, $D$ is defined by (1.4) and $A_{k i}, B_{k i}$ and $\widetilde{B}_{k i}$ are constants.

Lemma 2.2. For any integer $N \geq 0$ we have

$$
\begin{aligned}
c\|D u(t, \cdot)\|_{\Gamma, N, p, q} & \leq \sum_{|k| \leq N}\left\|D \Gamma^{k} u(t, \cdot)\right\|_{L^{p, q}\left(\mathbb{R}^{n}\right)} & \\
& \leq C\|D u(t, \cdot)\|_{\Gamma, N, p, q}, & \forall t \geq 0,
\end{aligned}
$$

where $1 \leq p, q \leq+\infty, c$ and $C$ are positive constants independent of $t$.

The following two lemmas follow directly from the chain rule and Hölder's inequality (cf. Li Ta-tsien and Zhou Yi [6]).

Lemma 2.3. For any integer $N \geq 0$,

$$
\begin{array}{r}
\|v(t, \cdot) w(t, \cdot)\|_{\Gamma, N, p_{0}, q_{0}, \chi} \leq C\left\{\|v(t, \cdot)\|_{\Gamma,[N / 2], p_{1}, q_{1}, \chi}\|w(t, \cdot)\|_{\Gamma, N, p_{2}, q_{2}, \chi}\right. \\
\left.+\|v(t, \cdot)\|_{\Gamma, N, p_{3}, q_{3}, \chi}\|w(t, \cdot)\|_{\Gamma,[N / 2], p_{4}, q_{4}, \chi}\right\}, \quad \forall t \geq 0
\end{array}
$$

and for any multi-index $k(|k| \leq N)$,

$$
\begin{aligned}
& \left\|\Gamma^{k} D(v(t, \cdot) w(t, \cdot))-v(t, \cdot) \Gamma^{k} D w(t, \cdot)\right\|_{p_{0}, q_{0}, \chi} \\
& \leq C\left\{\|D v(t, \cdot)\|_{\Gamma, N, p_{1}, q_{1}, \chi}\|w(t, \cdot)\|_{\Gamma,[N / 2]+1, p_{2}, q_{2}, \chi}\right. \\
& \left.\quad+\|v(t, \cdot)\|_{\Gamma,[N / 2]+1, p_{3}, q_{3}, \chi}\|w(t, \cdot)\|_{\Gamma, N, p_{4}, q_{4}, \chi}\right\}, \quad \forall t \geq 0,
\end{aligned}
$$

where [ ] stands for the integer part of a real number, $\chi(t, x)$ is the characteristic function of any given set in $\mathbb{R}_{+} \times \mathbb{R}^{n}, C$ is a positive constant and $1 \leq p_{i}, q_{i} \leq$ $+\infty(i=0,1, \ldots, 4)$ with

$$
\frac{1}{p_{0}}=\frac{1}{p_{1}}+\frac{1}{p_{2}}=\frac{1}{p_{3}}+\frac{1}{p_{4}}, \quad \frac{1}{q_{0}}=\frac{1}{q_{1}}+\frac{1}{q_{2}}=\frac{1}{q_{3}}+\frac{1}{q_{4}} .
$$


Lemma 2.4. Suppose that $F=F(w)$ is a sufficiently smooth function of $w=\left(w_{1}, \ldots, w_{M}\right)$ with

$$
F(w)=O\left(|w|^{1+\beta}\right), \quad \forall|w| \leq 1,
$$

where $\beta$ is an integer $\geq 0$. For any integer $N \geq 0$, if a vector function $w=w(t, x)$ satisfies

$$
\|w(t, \cdot)\|_{\Gamma,[N / 2], \infty} \leq 1, \quad \forall t \in[0, T],
$$

then, when $\beta=0$, we have

$$
\|F(w(t, \cdot))\|_{\Gamma, N, p, q, \chi} \leq C\|w(t, \cdot)\|_{\Gamma, N, p, q, \chi}, \quad \forall t \in[0, T] ;
$$

while, when $\beta \geq 1$, we have

$$
\begin{aligned}
& \|F(w(t, \cdot))\|_{\Gamma, N, p, q, \chi} \\
\leq & C\left(\prod_{i=1}^{\beta}\|w(t, \cdot)\|_{\Gamma,[N / 2], p_{i}, q_{i}, \chi}\right)\|w(t, \cdot)\|_{\Gamma, N, p_{0}, q_{0}, \chi}, \quad \forall t \in[0, T],
\end{aligned}
$$

where $1 \leq p, q, p_{i}, q_{i} \leq+\infty(i=0,1, \ldots, \beta)$ with

$$
\sum_{i=0}^{\beta} \frac{1}{p_{i}}=\frac{1}{p}, \quad \sum_{i=0}^{\beta} \frac{1}{q_{i}}=\frac{1}{q},
$$

$\chi(t, x)$ is the characteristic function of any given set in $\mathbb{R}_{+} \times \mathbb{R}^{n}$ and $C$ is a positive constant.

Lemma 2.5. For any integer $N \geq 0$, we have

$$
\sum_{|\alpha| \leq N}\left|D^{\alpha} u(t, x)\right| \leq C_{N}(1+|t-| x||)^{-N} \sum_{|k| \leq N}\left|\Gamma^{k} u(t, x)\right|,
$$

where $\alpha$ and $k$ are multi-indices and $C_{N}$ is a positive constant depending on $N$.

Proof. When $|t-| x|| \leq 1,(2.21)$ obviously holds. On the other hand, when $|t-| x|| \geq 1$, by (2.4)-(2.6) it is easy to see that

$$
\left\{\begin{array}{c}
\partial_{t}=\frac{t L_{0}-\sum_{i=1}^{n} x_{i} L_{i}}{t^{2}-|x|^{2}}, \\
\partial_{x_{j}}=\frac{t L_{j}-x_{j} L_{0}-\sum_{i=1}^{n} x_{i} \Omega_{i j}}{t^{2}-|x|^{2}}, \quad j=1, \ldots, n .
\end{array}\right.
$$


Thus, we have

$$
\begin{aligned}
\sum_{|\alpha|=1}\left|D^{\alpha} u(t, x)\right| & \leq C|t-| x||^{-1} \sum_{|k|=1}\left|\Gamma^{k} u(t, x)\right| \\
& \leq 2 C(1+|t-| x||)^{-1} \sum_{|k|=1}\left|\Gamma^{k} u(t, x)\right|,
\end{aligned}
$$

here and hereafter, in the proof $C$ always denotes a positive constant. Hence, noting (2.10), by induction we immediately get (2.21).

Lemma 2.6 (Sobolev embedding theorem on the ball). Let $B_{\lambda}$ be the ball in $\mathbb{R}^{n}$, centered at the origin and with the radius $\lambda>0$. For any given real number $p \geq 1$, we have

$$
\|u\|_{L^{\infty}\left(B_{\lambda}\right)} \leq C \lambda^{-n / p}\left(\sum_{|k| \leq S_{0}} \lambda^{|k|}\left\|D_{x}^{k} u\right\|_{L^{p}\left(B_{\lambda}\right)}\right),
$$

where $S_{0}=[n / p]+1$, and

$$
\|u\|_{L^{q}\left(B_{\lambda}\right)} \leq C \lambda^{-n(1 / p-1 / q)}\left(\sum_{|k| \leq S_{0}} \lambda^{|k|}\left\|D_{x}^{k} u\right\|_{L^{p}\left(B_{\lambda}\right)}\right),
$$

where $S_{0}$ is a positive integer and $q$ is determined as follows: if $S_{0}=n / p$, then $q \geq p$; while, if $S_{0}<n / p$, then $1 / q=1 / p-S_{0} / n$. In (2.23) and (2.24), $k$ are multi-indices and $C$ is a positive constant.

Proof. When $\lambda=1,(2.23)$ and (2.24) are the usual Sobolev embedding theorems on the unit ball. In the general case $\lambda>0$, the desired conclusion can be obtained by a scaling argument.

Lemma 2.7. Let $x=\left(x_{1}, x^{\prime}\right)$, where $x^{\prime}=\left(x_{2}, \ldots, x_{n}\right)$. We have

$$
\|f\|_{L^{\infty}\left(\mathbb{R} ; L^{2}\left(\mathbb{R}^{n-1}\right)\right)} \leq C\|f\|_{H^{S_{0}\left(\mathbb{R}^{n}\right)}}
$$

where $S_{0}>1 / 2$, and

$$
\|f\|_{L^{p}\left(\mathbb{R} ; L^{2}\left(\mathbb{R}^{n-1}\right)\right)} \leq C\|f\|_{H^{S_{0}\left(\mathbb{R}^{n}\right)}}
$$

where $0<S_{0}<1 / 2$ and $1 / p=1 / 2-S_{0}$. In (2.25) and (2.26), $C$ is a positive constant and $H^{S_{0}}\left(\mathbb{R}^{n}\right)$ is the Sobolev space equipped with the norm

$$
\|f\|_{H^{S_{0}\left(\mathbb{R}^{n}\right)}}=\left\|\left(1+|\xi|^{2}\right)^{S_{0} / 2} \hat{f}(\xi)\right\|_{L^{2}\left(\mathbb{R}^{n}\right)}
$$

where $\xi=\left(\xi_{1}, \ldots, \xi_{n}\right)$ and $\hat{f}$ is the Fourier transformation of $f$. 
Proof. By Sobolev embedding theorem and Parseval equality, we have

$$
\begin{aligned}
\|f\|_{L^{p}\left(\mathbb{R} ; L^{2}\left(\mathbb{R}^{n-1}\right)\right)} & =\left\|\int_{\mathbb{R}^{n-1}} f^{2}\left(\cdot, x^{\prime}\right) d x^{\prime}\right\|_{L^{p / 2}(\mathbb{R})}^{1 / 2} \\
& \leq\left(\int_{\mathbb{R}^{n-1}}\left\|f^{2}\left(\cdot, x^{\prime}\right)\right\|_{L^{p / 2}(\mathbb{R})} d x^{\prime}\right)^{1 / 2} \\
& =\|f\|_{L^{2}\left(\mathbb{R}^{n-1} ; L^{p}(\mathbb{R})\right)} \leq C\|f\|_{L^{2}\left(\mathbb{R}^{n-1} ; H^{\left.S_{0}(\mathbb{R})\right)}\right.} \\
& =C\left\|\left(1+\left|\xi_{1}\right|^{2}\right)^{S_{0} / 2} \hat{f}\left(\xi_{1}, \xi^{\prime}\right)\right\|_{L^{2}\left(\mathbb{R}^{n}\right)} \leq C\|f\|_{H^{S_{0}\left(\mathbb{R}^{n}\right)}} .
\end{aligned}
$$

This proves (2.26). The proof of (2.25) is similar.

Theorem 2.8. Suppose that $u=u(t, x)$ is a function with compact support in the variable $x$ for any fixed $t \geq 0$. For any integer $N \geq 0$, we have

$$
\begin{aligned}
& \|u(t, \cdot)\|_{\Gamma, N, \infty} \\
\leq & C(1+t)^{-(n-1) / p}(1+|t-| x||)^{-1 / p}\|u(t, \cdot)\|_{\Gamma, N+[n / p]+1, p}, \quad \forall t \geq 0,
\end{aligned}
$$

where $p \geq 1$ and $C$ is a positive constant.

Proof. See S. Klainerman [26].

Theorem 2.9. Let $\chi_{1}(t, x)$ be the characteristic function of the set $\{(t, x) \mid$ $|x| \leq(t+1) / 2\}$. For any integer $N \geq 0$ and any given $p \geq 1$, we have

$$
\|u(t, \cdot)\|_{\Gamma, N, \infty, \chi_{1}} \leq C(1+t)^{-n / p}\|u(t, \cdot)\|_{\Gamma, N+[n / p]+1, p, \chi_{1}}, \quad \forall t \geq 0,
$$

and

$$
\|u(t, \cdot)\|_{\Gamma, N, q, \chi_{1}} \leq C(1+t)^{-n(1 / p-1 / q)}\|u(t, \cdot)\|_{\Gamma, N+S_{0}, p, \chi_{1}}, \quad \forall t \geq 0
$$

where $C$ is a positive constant, $S_{0}$ is a positive integer and $q$ is determined as follows: if $S_{0}=n / p$, then $q \geq p$; while, if $S_{0}<n / p$, then $1 / q=1 / p-S_{0} / n$.

Proof. Using Lemma 2.6 (in which we take $\lambda=(t+1) / 2$ ) and Lemma 2.5, we get (2.29) and (2.30) for $N=0$, then (2.29) and (2.30) hold for any integer $N \geq 0$. 
Theorem 2.10. Let $\chi_{2}(x)$ be the characteristic function of the set $\{x \mid$ $|x| \geq a\}$. We have

$$
\left\|\chi_{2} f\right\|_{L^{\infty, 2}\left(\mathbb{R}^{n}\right)} \leq C a^{-\left(n / 2-S_{0}\right)}\|f\|_{\dot{H}^{s_{0}\left(\mathbb{R}^{n}\right)}},
$$

where $1 / 2<S_{0}<n / 2$, and for any given $p>2$ we have

$$
\left\|\chi_{2} f\right\|_{L^{p, 2}\left(\mathbb{R}^{n}\right)} \leq C a^{-(n-1) S_{0}}\|f\|_{\dot{H}^{S_{0}\left(\mathbb{R}^{n}\right)}},
$$

where $S_{0}=1 / 2-1 / p$. In (2.31) and (2.32), $C$ is a positive constant and $\dot{H}^{S_{0}}\left(\mathbb{R}^{n}\right)$ stands for the homogeneous Sobolev space equipped with the norm

$$
\|f\|_{\dot{H}^{S_{0}\left(\mathbb{R}^{n}\right)}}=\left\||\xi|^{S_{0}} \hat{f}(\xi)\right\|_{L^{2}\left(\mathbb{R}^{n}\right)},
$$

where $\hat{f}(\xi)$ is the Fourier transformation of $f(x)$.

Proof. We first prove (2.32). Without loss of generality, we may suppose that $a=4$. In the general case $a>0,(2.32)$ can be obtained by a scaling argument.

Let $\Phi(x)=\Phi(|x|) \in C_{0}^{\infty}\left(\mathbb{R}^{n}\right)$ such that

$$
\operatorname{supp} \Phi \subseteq\{x|1 \leq| x \mid \leq 3\}
$$

and

$$
\sum_{j=0}^{\infty} \Phi_{j}(x) \equiv 1,
$$

where

$$
\Phi_{j}(x)=\Phi\left(2^{-j} x\right) .
$$

Let $g=\Phi f$. Taking a suitable homeomorphic transformation of variables and then, using (2.25), it is easy to see that if $1 / 2<S_{0}<n / 2$, then

$$
\|g\|_{L^{\infty, 2}\left(\mathbb{R}^{n}\right)} \leq C\|g\|_{H^{S_{0}\left(\mathbb{R}^{n}\right)}} .
$$

By Poincaré's inequality and Parseval's equality, we have

$$
\begin{aligned}
\|g\|_{H^{S_{0}\left(\mathbb{R}^{n}\right)}} \leq & C\|g\|_{\dot{H}^{S_{0}\left(\mathbb{R}^{n}\right)}}=C\left\||\xi|^{S_{0}} \hat{g}(\xi)\right\|_{L^{2}\left(\mathbb{R}^{n}\right)} \\
& =C\left\||\xi|^{S_{0}} \int_{\mathbb{R}^{n}} \hat{\Phi}(\xi-\eta) \hat{f}(\eta) d \eta\right\|_{L^{2}\left(\mathbb{R}^{n}\right)} \\
& \leq C\left(\left\|\int_{\mathbb{R}^{n}}|\xi-\eta|^{S_{0}}|\hat{\Phi}(\xi-\eta) \hat{f}(\eta)| d \eta\right\|_{L^{2}\left(\mathbb{R}^{n}\right)}\right. \\
& \left.\quad+\left\|\int_{\mathbb{R}^{n}}|\hat{\Phi}(\xi-\eta)||\eta|^{S_{0}}|\hat{f}(\eta)| d \eta\right\|_{L^{2}\left(\mathbb{R}^{n}\right)}\right) \\
& =C\left(\left\|\Phi^{*} f_{*}\right\|_{L^{2}\left(\mathbb{R}^{n}\right)}+\left\|\Phi_{*} f^{*}\right\|_{L^{2}\left(\mathbb{R}^{n}\right)}\right) \\
& \leq C\left(\left\|f_{*}\right\|_{L^{\gamma}\left(\mathbb{R}^{n}\right)}\left\|\Phi^{*}\right\|_{L^{n / S_{0}\left(\mathbb{R}^{n}\right)}}+\left\|\Phi_{*}\right\|_{L^{\infty}\left(\mathbb{R}^{n}\right)}\left\|f^{*}\right\|_{L^{2}\left(\mathbb{R}^{n}\right)}\right),
\end{aligned}
$$


where

$$
\left\{\begin{array}{l}
\hat{\Phi}^{*}(\xi)=|\xi|^{S_{0}}|\hat{\Phi}(\xi)|, \\
\hat{f}_{*}(\xi)=|\hat{f}(\xi)|, \\
\hat{\Phi}_{*}(\xi)=|\hat{\Phi}(\xi)|, \\
\hat{f}^{*}(\xi)=|\xi|^{S_{0}}|\hat{f}(\xi)|
\end{array}\right.
$$

and $1 / \gamma=1 / 2-S_{0} / n$. By the Sobolev embedding theorem,

$$
\left\|f_{*}\right\|_{L^{\gamma}\left(\mathbb{R}^{n}\right)} \leq C\left\|f_{*}\right\|_{\dot{H}^{S_{0}\left(\mathbb{R}^{n}\right)}}=C\|f\|_{\dot{H}^{S_{0}\left(\mathbb{R}^{n}\right)}}
$$

and by Parseval's equality,

$$
\left\|f^{*}\right\|_{L^{2}\left(\mathbb{R}^{n}\right)}=\|f\|_{\dot{H}^{S_{0}\left(\mathbb{R}^{n}\right)}} .
$$

Noting that $1 / 2<S_{0}<n / 2$, by the Hausdorff-Young inequality, we have

$$
\begin{aligned}
\left\|\Phi^{*}\right\|_{L^{n / S_{0}\left(\mathbb{R}^{n}\right)}} & \leq C\left\|\hat{\Phi}^{*}\right\|_{L^{n /\left(n-S_{0}\right)\left(\mathbb{R}^{n}\right)}} \\
& =C\left\||\xi|^{S_{0}} \hat{\Phi}(\xi)\right\|_{L^{n /\left(n-S_{0}\right)\left(\mathbb{R}^{n}\right)}}<+\infty .
\end{aligned}
$$

Moreover, it is easy to see that

$$
\left\|\Phi_{*}\right\|_{L^{\infty}\left(\mathbb{R}^{n}\right)} \leq C\|\hat{\Phi}\|_{L^{1}\left(\mathbb{R}^{n}\right)}<+\infty .
$$

The combination of (2.37)-(2.38) and (2.40)-(2.43) gives

$$
\|\Phi f\|_{L^{\infty, 2}\left(\mathbb{R}^{n}\right)} \leq C\|f\|_{\dot{H}^{S_{0}\left(\mathbb{R}^{n}\right)}},
$$

then a scaling argument yields

$$
\left\|\Phi_{j} f\right\|_{L^{\infty, 2}\left(\mathbb{R}^{n}\right)} \leq C 2^{j\left(S_{0}-n / 2\right)}\|f\|_{\dot{H}^{S_{0}\left(\mathbb{R}^{n}\right)}} .
$$

Hence

$$
\begin{aligned}
\left\|\chi_{2} f\right\|_{L^{\infty, 2}\left(\mathbb{R}^{n}\right)} & \leq \sum_{j=0}^{\infty}\left\|\Phi_{j} f\right\|_{L^{\infty, 2}\left(\mathbb{R}^{n}\right)} \\
& \leq C \sum_{j=0}^{\infty} 2^{j\left(S_{0}-n / 2\right)}\|f\|_{\dot{H}^{S_{0}\left(\mathbb{R}^{n}\right)}} \\
& \leq C\|f\|_{\dot{H}^{S_{0}\left(\mathbb{R}^{n}\right)}} .
\end{aligned}
$$

This is just (2.32) in the case $a=4$. (2.32) is then proved.

Using $(2.26),(2.31)$ can be proved in a completely similar way. 
Theorem 2.11. Let $\chi_{2}(x)$ be the characteristic function of the set $\{x \mid$ $|x| \geq a\}$ and $\chi_{1}=1-\chi_{2}$. Then

$$
\|f\|_{\dot{H}^{-S_{0}\left(\mathbb{R}^{n}\right)}} \leq C\left(\left\|\chi_{1} f\right\|_{L^{q}\left(\mathbb{R}^{n}\right)}+a^{-\left(n / 2-S_{0}\right)}\left\|\chi_{2} f\right\|_{L^{1,2}\left(\mathbb{R}^{n}\right)}\right),
$$

where

$$
\frac{1}{2}<S_{0}<\frac{n}{2} \quad \text { and } \quad \frac{1}{q}=\frac{1}{2}+\frac{S_{0}}{n} .
$$

Proof. By definition,

$$
\|f\|_{\dot{H}^{-S_{0}\left(\mathbb{R}^{n}\right)}}=\sup _{\substack{v \in \dot{H}^{S_{0}\left(\mathbb{R}^{n}\right)} \\ v \neq 0}} \frac{\int f v}{\|v\|_{\dot{H}^{S_{0}\left(\mathbb{R}^{n}\right)}}} .
$$

Using Hölder's inequality, we have

$$
\begin{aligned}
\int f v & =\int\left(\chi_{1} f\right) v+\int\left(\chi_{2} f\right) v \\
& \leq\left\|\chi_{1} f\right\|_{L^{q}\left(\mathbb{R}^{n}\right)}\|v\|_{L^{\gamma}\left(\mathbb{R}^{n}\right)}+\left\|\chi_{2} f\right\|_{L^{1,2}\left(\mathbb{R}^{n}\right)}\left\|\chi_{2} v\right\|_{L^{\infty, 2}\left(\mathbb{R}^{n}\right)},
\end{aligned}
$$

where $q$ is defined by $(2.48)$ and $1 / \gamma=1 / 2-S_{0} / n$.

By the Sobolev embedding theorem,

$$
\|v\|_{L^{\gamma}\left(\mathbb{R}^{n}\right)} \leq C\|v\|_{\dot{H}^{S_{0}\left(\mathbb{R}^{n}\right)}}
$$

and, using (2.31), we have

$$
\left\|\chi_{2} v\right\|_{L^{\infty, 2}\left(\mathbb{R}^{n}\right)} \leq C a^{-\left(n / 2-S_{0}\right)}\|v\|_{\dot{H}^{S_{0}\left(\mathbb{R}^{n}\right)}} .
$$

Substituting (2.50)-(2.52) into (2.49) yields the desired estimate (2.47).

\section{Some estimates on solutions to linear wave equations.}

Lemma 3.1. Let $u=u(t, x)$ be the solution to the following Cauchy problem for linear wave equations:

$$
\begin{array}{lr}
\square u(t, x)=0, & (t, x) \in \mathbb{R}_{+} \times \mathbb{R}^{n}, \\
t=0: u=f(x), u_{t}=g(x), & x \in \mathbb{R}^{n},
\end{array}
$$

with

$$
\operatorname{supp}\{f, g\} \subseteq\{x|| x \mid \leq \rho\},
$$

where $\rho$ is a positive constant. Then, when $t-|x| \geq 2 \rho$, we have

$$
\begin{aligned}
|u(t, x)| \leq C(t+|x|)^{-(n-1) / 2}(t-|x|)^{-(n-1) / 2} & \\
\cdot & \left\{(t-|x|)^{-1}\|f\|_{L^{1}\left(\mathbb{R}^{n}\right)}+\|g\|_{L^{1}\left(\mathbb{R}^{n}\right)}\right\},
\end{aligned}
$$

where $C$ is a positive constant independent of $\rho$. 
Proof. The solution to Cauchy problem (3.1)-(3.2) can be explicitly expressed by

$$
u=\frac{\partial}{\partial t} E_{t} * f+E_{t} * g \triangleq u_{1}+u_{2},
$$

where

$$
E_{t}(x)=2^{-1} \pi^{(1-n) / 2} \chi_{+}^{(1-n) / 2}\left(t^{2}-|x|^{2}\right)
$$

and $\chi_{+}^{a}$ is defined as follows: when $a>0$,

$$
\chi_{+}^{a}(A)= \begin{cases}A^{a} / \Gamma(a+1), & \text { if } A>0, \\ 0, & \text { if } A \leq 0,\end{cases}
$$

then

$$
\frac{d \chi_{+}^{a+1}(A)}{d A}=\chi_{+}^{a}(A)
$$

while, when $a \leq 0, \chi_{+}^{a}(A)$ can be defined by (3.8) in the sense of distribution. It is easy to see that $\chi_{+}^{a}(A)$ is a homogeneous distribution of degree $a$ and

$$
\text { sing supp } \chi_{+}^{a} \subseteq\{A \mid A \leq 0\} .
$$

We first consider

$$
u_{2}(t, x)=\left(E_{t} * g\right)(t, x)=C_{0}\left\langle\chi_{+}^{(1-n) / 2}\left(t^{2}-|x-y|^{2}\right), g(y)\right\rangle,
$$

where $C_{0}=2^{-1} \pi^{(1-n) / 2}$.

Noting that $t-|x| \geq 2 \rho$, if $|y| \leq \rho$, then it is easy to see that

$$
\begin{aligned}
t^{2}-|x-y|^{2} & =t^{2}-|x|^{2}+2 x \cdot y-|y|^{2} \\
& \geq\left(t^{2}-|x|^{2}\right)\left(1-\frac{2 \rho|x|+\rho^{2}}{(t-|x|)(t+|x|)}\right) \\
& \geq \frac{1}{2}\left(t^{2}-|x|^{2}\right)>0 .
\end{aligned}
$$

Hence, noting that $\chi_{+}^{a}(A)$ is a homogeneous distribution of degree $a$ and using (3.3), under the hypothesis $t-|x| \geq 2 \rho$ we have

$$
\begin{aligned}
\left|u_{2}(t, x)\right| & \leq C_{0} \chi_{+}^{(1-n) / 2}(1) \int_{\mathbb{R}^{n}}\left(t^{2}-|x-y|^{2}\right)^{(1-n) / 2}|g(y)| d y \\
& \leq \tilde{C}(t+|x|)^{-(n-1) / 2}(t-|x|)^{-(n-1) / 2}\|g\|_{L^{1}\left(\mathbb{R}^{n}\right)},
\end{aligned}
$$


here and hereafter $\tilde{C}$ denotes a positive constant independent of $\rho$.

On the other hand, noting (3.8) we have

$$
\begin{aligned}
u_{1}(t, x) & =\left(\frac{\partial}{\partial t} E_{t} * f\right)(t, x) \\
& =2 C_{0} t\left\langle\chi_{+}^{-(n+1) / 2}\left(t^{2}-|x-y|^{2}\right), f(y)\right\rangle,
\end{aligned}
$$

then similarly we get

$$
\begin{aligned}
\left|u_{1}(t, x)\right| & \leq \tilde{C} t(t+|x|)^{-(n+1) / 2}(t-|x|)^{-(n+1) / 2}\|f\|_{L^{1}\left(\mathbb{R}^{n}\right)} \\
& \leq \tilde{C}(t+|x|)^{-(n-1) / 2}(t-|x|)^{-(n+1) / 2}\|f\|_{L^{1}\left(\mathbb{R}^{n}\right)} .
\end{aligned}
$$

(3.4) follows immediately from (3.12) and (3.14).

Theorem 3.2. Let $u=u(t, x)$ be the solution to Cauchy problem (3.1)(3.2). For any integer $N \geq 0$, we have

$$
\begin{aligned}
\|u(t, \cdot)\|_{\Gamma, N, \infty} \leq C(1+t)^{-(n-1) / 2}\left(\|u(0, \cdot)\|_{\Gamma, N+n, 1}\right. & \\
\left.+\left\|u_{t}(0, \cdot)\right\|_{\Gamma, N+n-1,1}\right), & \forall t \geq 0,
\end{aligned}
$$

where $C$ is a positive constant and $\|u(0, \cdot)\|_{\Gamma, N+n, 1}$ denotes the value of $\|u(t, \cdot)\|_{\Gamma, N+n, 1}$ at $t=0$, etc.

Proof. By S. Klainerman [27],

$$
\begin{array}{r}
|u(t, x)| \leq C(1+t)^{-(n-1) / 2}\left(\|f\|_{W^{n, 1}\left(\mathbb{R}^{n}\right)}+\|g\|_{W^{n-1,1}\left(\mathbb{R}^{n}\right)}\right), \\
\forall t \geq 0, \forall x \in \mathbb{R}^{n},
\end{array}
$$

then using Lemma 2.1 we immediately obtain (3.15).

Lemma 3.3. Let $u=u(t, x)$ be the solution to Cauchy problem (3.1)-(3.2) with (3.3) (in which $\rho=1)$. For any integer $N \geq 0$ and any multi-index $k$ $(|k| \leq N)$, we have

$$
\begin{aligned}
& \left|\Gamma^{k} u(t, x)\right| \leq C(1+t+|x|)^{-(n-1) / 2}(1+|t-| x||)^{-(n-1) / 2} \\
& \quad \cdot\left(\|u(0, \cdot)\|_{\Gamma, N+n, 1}+\left\|u_{t}(0, \cdot)\right\|_{\Gamma, N+n-1,1}\right), \quad \forall t \geq 0, \forall x \in \mathbb{R}^{n}
\end{aligned}
$$

where $C$ is a positive constant. 
Proof. By Lemma 2.1 it suffices to prove that

$$
\begin{aligned}
& |u(t, x)| \leq C(1+t+|x|)^{-(n-1) / 2}(1+|t-| x||)^{-(n-1) / 2} \\
& \quad\left(\|f\|_{W^{n, 1}\left(\mathbb{R}^{n}\right)}+\|g\|_{W^{n-1,1}\left(\mathbb{R}^{n}\right)}\right), \quad \forall t \geq 0, \forall x \in \mathbb{R}^{n} .
\end{aligned}
$$

When $t-|x| \geq 2$, (3.18) easily follows from (3.4); while, when $t-|x| \leq 2$, noting that supp $u \subseteq\{(t, x)|| x \mid \leq t+1\}$, (3.18) comes from (3.16).

Theorem 3.4. Suppose that $n \geq 3$. Let $u=u(t, x)$ be the solution to the following inhomogeneous wave equation:

$$
\square u=F(t, x), \quad(t, x) \in \mathbb{R}_{+} \times \mathbb{R}^{n}
$$

with the initial data (3.2). For any integer $N \geq 0$, we have

$$
\begin{aligned}
& \|u(t, \cdot)\|_{\Gamma, N, 2} \leq C\left\{\|u(0, \cdot)\|_{\Gamma, N, 2}+\left\|u_{t}(0, \cdot)\right\|_{\Gamma, N, q}\right. \\
+ & \left.\int_{0}^{t}\left[\|F(\tau, \cdot)\|_{\Gamma, N, q, \chi_{1}}+(1+\tau)^{-(n-2) / 2}\|F(\tau, \cdot)\|_{\Gamma, N, 1,2, \chi_{2}}\right] d \tau\right\}, \quad \forall t \geq 0,
\end{aligned}
$$

where $\chi_{1}$ is the characteristic function of the set $\{(t, x)|| x \mid \leq(t+1) / 2\}, \chi_{2}=$ $1-\chi_{1}$,

$$
\frac{1}{q}=\frac{1}{2}+\frac{1}{n}
$$

and $C$ is a positive constant.

Proof. cf. Lemma 2.5 in Li Ta-tsien and Yu Xin [5].

Theorem 3.5. Suppose that $n \geq 3$. Under the assumptions of Theorem 3.4, suppose, furthermore, that (3.3) (in which $\rho=1$ ) holds and

$$
\operatorname{supp} F \subseteq\{(t, x)|| x \mid \leq t+1\} .
$$

Then, for any integer $N \geq 0$ we have

$$
\begin{aligned}
& \|u(t, \cdot)\|_{\Gamma, N, 2, \chi_{1}} \leq C(1+t)^{-(n-2) / 2}\left\{\|u(0, \cdot)\|_{\Gamma, N, 2}+\left\|u_{t}(0, \cdot)\right\|_{\Gamma, N, q}\right. \\
& \left.+\int_{0}^{t}\left[(1+\tau)^{(n-2) / 2}\|F(\tau, \cdot)\|_{\Gamma, N, q, \chi_{1}}+\|F(\tau, \cdot)\|_{\Gamma, N, 1,2, \chi_{2}}\right] d \tau\right\}, \quad \forall t \geq 0,
\end{aligned}
$$

where $C$ is a positive constant. 
Proof. By Lemma 2.1, it is only necessary to prove that

$$
\begin{aligned}
& \|u(t, \cdot)\|_{2, \chi_{1}} \leq C(1+t)^{-(n-2) / 2}\left\{\|f\|_{L^{2}\left(\mathbb{R}^{n}\right)}+\|g\|_{L^{q}\left(\mathbb{R}^{n}\right)}\right. \\
& \left.\quad+\int_{0}^{t}\left[(1+\tau)^{(n-2) / 2}\|F(\tau, \cdot)\|_{q, \chi_{1}}+\|F(\tau, \cdot)\|_{1,2, \chi_{2}}\right] d \tau\right\}, \quad \forall t \geq 0 .
\end{aligned}
$$

We first consider the case $F \equiv 0$. When $t \leq 5$, by (3.30) we have

$$
\|u(t, \cdot)\|_{2, \chi_{1}} \leq C(1+t)^{-(n-2) / 2}\left(\|f\|_{L^{2}\left(\mathbb{R}^{n}\right)}+\|g\|_{L^{q}\left(\mathbb{R}^{n}\right)}\right) ;
$$

while, when $t \geq 5,|x| \leq(t+1) / 2$ implies $t-|x| \geq 2$, then, using Hölder's inequality and noting (3.3), it follows from (3.4) that

$$
|u(t, x)| \leq C(t+|x|)^{-(n-1) / 2}(t-|x|)^{-(n-1) / 2}\left(\|f\|_{L^{2}\left(\mathbb{R}^{n}\right)}+\|g\|_{L^{q}\left(\mathbb{R}^{n}\right)}\right),
$$

hence, by integration it is easy to see that (3.25) still holds. Thus, (3.24) is valid in the case $F \equiv 0$.

By the superposition principle it remains to consider the case $f \equiv g \equiv 0$.

For any given $t \geq 0$, if

$$
\operatorname{supp} F(\tau, \cdot) \subseteq\{\tau \mid 6 \tau+5 \geq t\}
$$

then, by (3.20), it is easily seen that

$$
\begin{aligned}
& \leq C \int_{\max (0,(t-5) / 6)}^{t}\left[\|F(\tau, \cdot)\|_{q, \chi_{1}}+(1+\tau)^{-(n-2) / 2}\|F(\tau, \cdot)\|_{1,2, \chi_{2}}\right] d \tau \\
& \leq C(1+t)^{-(n-2) / 2} \int_{\max (0,(t-5) / 6)}^{t}\left[(1+\tau)^{(n-2) / 2}\|F(\tau, \cdot)\|_{q, \chi_{1}}\right. \\
& \left.\quad+\|F(\tau, \cdot)\|_{1,2, \chi_{2}}\right] d \tau \\
& \leq C(1+t)^{-(n-2) / 2} \int_{0}^{t}\left[(1+\tau)^{(n-2) / 2}\|F(\tau, \cdot)\|_{q, \chi_{1}}+\|F(\tau, \cdot)\|_{1,2, \chi_{2}}\right] d \tau .
\end{aligned}
$$

On the other hand, if

$$
\operatorname{supp} F(\tau, \cdot) \subseteq\{\tau \mid 6 \tau+5 \leq t\},
$$

then, noting that when $|x| \leq(t+1) / 2$, we have

$$
t-\tau-|x| \geq \frac{t+1}{3} \geq 2(\tau+1)
$$


by Duhamel's principle and using Lemma 3.1, it is easy to see that

$$
\left|\chi_{1} u(t, x)\right| \leq C(1+t)^{-(n-1)} \int_{0}^{t}\|F(\tau, \cdot)\|_{L^{1}\left(\mathbb{R}^{n}\right)} d \tau .
$$

By (3.22) and using Hölder's inequality, we have

$$
\|F(\tau, \cdot)\|_{L^{1}\left(\mathbb{R}^{n}\right)} \leq C\left((1+\tau)^{(n-2) / 2}\|F(\tau, \cdot)\|_{q, \chi_{1}}+\|F(\tau, \cdot)\|_{1,2, \chi_{2}}\right) .
$$

Moreover, it is easy to see that

$$
\left\|\chi_{1} u(t, \cdot)\right\|_{L^{2}\left(\mathbb{R}^{n}\right)} \leq C(1+t)^{n / 2}\left\|\chi_{1} u(t, \cdot)\right\|_{L^{\infty}\left(\mathbb{R}^{n}\right)} .
$$

The combination of (3.30)-(3.32) shows that (3.27) still holds. This proves (3.24) in the case $f \equiv g \equiv 0$.

Theorem 3.6. Suppose that $n \geq 2$. Under the assumptions of Theorem 3.4, for any integer $N \geq 0$ we have

$$
\begin{aligned}
& \|u(t, \cdot)\|_{\Gamma, N, p, 2, \chi_{2}} \\
& \leq C(1+t)^{-(n-1)(1 / 2-1 / p)}\left\{\sum_{|k| \leq N}\left\|\Gamma^{k} u(0, \cdot)\right\|_{\dot{H}^{S_{0}}\left(\mathbb{R}^{n}\right)}\right. \\
& \quad+\left\|u_{t}(0, \cdot)\right\|_{\Gamma, N, \gamma}+\int_{0}^{t}\left[\|F(\tau, \cdot)\|_{\Gamma, N, \gamma, \chi_{1}}\right. \\
& \left.\left.\quad+(1+\tau)^{-\left((n-2) / 2+S_{0}\right)}\|F(\tau, \cdot)\|_{\Gamma, N, 1,2, \chi_{2}}\right] d \tau\right\}, \quad \forall t \geq 0,
\end{aligned}
$$

where

$$
p>2, \quad S_{0}=\frac{1}{2}-\frac{1}{p}, \quad \frac{1}{\gamma}=\frac{1}{2}+\frac{1-S_{0}}{n},
$$

$\|\cdot\|_{\dot{H}^{S_{0}\left(\mathbb{R}^{n}\right)}}$ is defined by (2.33) and $C$ is a positive constant.

Proof. By Lemma 2.1, it suffices to prove that

$$
\begin{aligned}
& \|u(t, \cdot)\|_{p, 2, \chi_{2}} \leq C(1+t)^{-(n-1)(1 / 2-1 / p)}\left\{\|f\|_{\dot{H}^{S_{0}\left(\mathbb{R}^{n}\right)}}+\|g\|_{L^{\gamma}\left(\mathbb{R}^{n}\right)}\right. \\
& \left.+\int_{0}^{t}\left[\|F(\tau, \cdot)\|_{\gamma, \chi_{1}}+(1+\tau)^{-\left((n-2) / 2+S_{0}\right)}\|F(\tau, \cdot)\|_{1,2, \chi_{2}}\right] d \tau\right\}, \quad \forall t \geq 0 .
\end{aligned}
$$


By (2.32) we have

$$
\|u(t, \cdot)\|_{p, 2, \chi_{2}} \leq C(1+t)^{-(n-1)(1 / 2-1 / p)}\|u(t, \cdot)\|_{\dot{H}^{S_{0}\left(\mathbb{R}^{n}\right)}} .
$$

Taking the Fourier transformation, it follows from (3.19) and (3.2) that

$$
\begin{aligned}
& \frac{\partial^{2} \hat{u}(t, \xi)}{\partial t^{2}}+|\xi|^{2} \hat{u}(t, \xi)=\hat{F}(t, \xi), \\
& t=0: \hat{u}=\hat{f}(\xi), \hat{u}_{t}=\hat{g}(\xi) .
\end{aligned}
$$

It is easy to see that

$$
\hat{u}(t, \xi)=\cos (|\xi| t) \hat{f}(\xi)+\frac{\sin (|\xi| t)}{|\xi|} \hat{g}(\xi)+\int_{0}^{t} \frac{\sin (|\xi|(t-\tau))}{|\xi|} \hat{F}(\tau, \xi) d \tau,
$$

hence

$$
|\hat{u}(t, \xi)| \leq|\hat{f}(\xi)|+|\xi|^{-1}|\hat{g}(\xi)|+\int_{0}^{t}|\xi|^{-1}|\hat{F}(\tau, \xi)| d \tau,
$$

then, by (2.33), we get

$$
\begin{aligned}
\|u(t, \cdot)\|_{\dot{H}^{S_{0}\left(\mathbb{R}^{n}\right)}} \leq\|f\|_{\dot{H}^{S_{0}\left(\mathbb{R}^{n}\right)}}+\|g\|_{\dot{H}^{S_{0}-1}\left(\mathbb{R}^{n}\right)} \\
\quad+\int_{0}^{t}\|F(\tau, \cdot)\|_{\dot{H}^{S_{0}-1}\left(\mathbb{R}^{n}\right)} d \tau .
\end{aligned}
$$

By the Sobolev embedding theorem we have

$$
\|g\|_{\dot{H}^{S_{0}-1}\left(\mathbb{R}^{n}\right)} \leq C\|g\|_{L^{\gamma}\left(\mathbb{R}^{n}\right)} .
$$

Moreover, by Theorem 2.11,

$$
\begin{aligned}
& \|F(\tau, \cdot)\|_{\dot{H}^{S_{0}-1}\left(\mathbb{R}^{n}\right)} \\
\leq & C\left(\|F(\tau, \cdot)\|_{\gamma, \chi_{1}}+(1+\tau)^{-\left((n-2) / 2+S_{0}\right)}\|F(\tau, \cdot)\|_{1,2, \chi_{2}}\right) .
\end{aligned}
$$

Combining (3.36) and (3.41)-(3.43) yields (3.35).

Lemma 3.7. Let $u=u(t, x)$ be the solution to equation (3.19) with zero initial data. Suppose that $n$ is an even number $\geq 2$, then

$$
\begin{aligned}
& |u(t, x)| \leq C(1+t+|x|)^{-(n-1) / 2}(1+|t-| x||)^{-\ell} \\
& \cdot \int_{0}^{t}(1+\tau)^{\ell-(n-1) / 2}\|F(\tau, \cdot)\|_{\Gamma, n-1,1} d \tau,
\end{aligned}
$$

where $0 \leq \ell \leq(n-1) / 2$. 
Proof. See L. Hörmander [28].

Lemma 3.8. Suppose that $n=4$. Suppose furthermore that $u=u(t, x)$ satisfies equation (3.19) with (3.22) and the zero initial condition. Then we have

$$
|u(t, x)| \leq C(1+t+|x|)^{-3 / 2}(1+|t-| x||)^{-1 / 2} \sup _{0 \leq \tau \leq t}\|F(\tau, \cdot)\|_{\Gamma, 3,1},
$$

where $C$ is a positive constant.

Proof. Let $\varphi \in C_{0}^{\infty}(\mathbb{R})$ such that

$$
\varphi(\eta)= \begin{cases}1, & \text { if } \eta \geq 2 \\ 0, & \text { if } \eta \leq 1\end{cases}
$$

For any given $(t, x) \in \mathbb{R}_{+} \times \mathbb{R}^{n}$, let

$$
u=u_{1}+u_{2},
$$

where $u_{1}=u_{1}(s, y)$ and $u_{2}=u_{2}(s, y)$ satisfy

$$
\square u_{1}(s, y)=\varphi\left(\frac{s}{2+|t-| x||}\right) F(s, y)
$$

and

$$
\square u_{2}(s, y)=\left(1-\varphi\left(\frac{s}{2+|t-| x||}\right)\right) F(s, y),
$$

respectively, and the zero initial condition.

In order to estimate $u_{1}$, using Lemma 3.7 (in which we take $n=4$ and $\ell=0$ ) and noting (3.46), when $s \leq 2+|t-| x||$, we have

$$
\left|u_{1}(s, y)\right|=0
$$

while, when $s \geq 2+|t-| x||$, we have

$$
\begin{aligned}
\left|u_{1}(s, y)\right| & \leq C(1+s+|y|)^{-3 / 2} \int_{2+|t-| x||}^{s}(1+\tau)^{-3 / 2}\|F(\tau, \cdot)\|_{\Gamma, 3,1} d \tau \\
& \leq C(1+s+|y|)^{-3 / 2}(1+|t-| x||)^{-1 / 2} \sup _{0 \leq \tau \leq s}\|F(\tau, \cdot)\|_{\Gamma, 3,1} .
\end{aligned}
$$


In particular, taking $(s, y)=(t, x)$ we get

$$
\left|u_{1}(t, x)\right| \leq C(1+t+|x|)^{-3 / 2}(1+|t-| x||)^{-1 / 2} \sup _{0 \leq \tau \leq t}\|F(\tau, \cdot)\|_{\Gamma, 3,1} .
$$

Still using Lemma 3.7 (in which we take $n=4$ and $\ell=\frac{3}{2}$ ), similarly we get

$$
\begin{aligned}
\left|u_{2}(s, y)\right| & \leq C(1+s+|y|)^{-3 / 2}(1+|s-| y||)^{-3 / 2} \int_{0}^{2(2+|t-| x||)}\|F(\tau, \cdot)\|_{\Gamma, 3,1} d \tau \\
& \leq C(1+s+|y|)^{-3 / 2}(1+|s-| y||)^{-3 / 2}(1+|t-| x||) \sup _{0 \leq \tau \leq s}\|F(\tau, \cdot)\|_{\Gamma, 3,1},
\end{aligned}
$$

then, taking $(s, y)=(t, x)$ we obtain

$$
\left|u_{2}(t, x)\right| \leq C(1+t+|x|)^{-3 / 2}(1+|t-| x||)^{-1 / 2} \sup _{0 \leq \tau \leq t}\|F(\tau, \cdot)\|_{\Gamma, 3,1}
$$

The combination of (3.50) and (3.51) leads to (3.45).

Combining Lemma 3.3 and Lemma 3.8 and noting Lemma 2.1, we get:

Theorem 3.9. Suppose that $n=4$. Let $u=u(t, x)$ be the solution to Cauchy problem (3.19) and (3.2) with (3.3) (in which $\rho=1)$ and (3.22). For any integer $N \geq 0$ and any multi-index $k(|k| \leq N)$, we have

$$
\begin{aligned}
\left|\Gamma^{k} u(t, x)\right| \leq & C(1+t+|x|)^{-3 / 2}(1+|t-| x||)^{-3 / 2}\left\{\|u(0, \cdot)\|_{\Gamma, N+4,1}\right. \\
& \left.+\left\|u_{t}(0, \cdot)\right\|_{\Gamma, N+3,1}+(1+|t-| x||) \sup _{0 \leq \tau \leq t}\|F(\tau, \cdot)\|_{\Gamma, N+3,1}\right\}
\end{aligned}
$$

where $C$ is a positive constant.

Lemma 3.10. Suppose that $n=4$. Under the assumptions of Lemma 3.8, we have

$$
\begin{aligned}
& \left\|(1+|t-| \cdot||)^{-1 / 2} u(t, \cdot)\right\|_{1,2, \chi_{2}} \leq C(1+t)^{3 / 2} \\
& \cdot \int_{0}^{t}\left[(1+\tau)^{1 / 3}\|F(\tau, \cdot)\|_{3 / 2, \chi_{1}}+(1+\tau)^{-1}\|F(\tau, \cdot)\|_{1,2, \chi_{2}}\right] d \tau, \quad \forall t \geq 0,
\end{aligned}
$$

where $\chi_{1}$ is the characteristic function of the set $\{(t, x)|| x \mid \leq(t+1) / 2\}, \chi_{2}=$ $1-\chi_{1}$ and $C$ is a positive constant. 
Proof. Since the wave has a finite speed of propagation, we have

$$
\text { supp } u \subseteq\{(t, x)|| x \mid \leq t+1\} .
$$

Let $\varphi$ be the characteristic function of the set $\{(t, x)|| x \mid \leq t+1\}$. By Hölder's inequality we have

$$
\left\|(1+|t-| \cdot||)^{-1 / 2} u(t, \cdot)\right\|_{1,2, \chi_{2}} \leq\left\|(1+|t-| \cdot||)^{-1 / 2}\right\|_{6 / 5, \infty, \varphi}\|u(t, \cdot)\|_{6,2, \chi_{2}} .
$$

By $(2.7)-(2.8)$, it is easy to see that

$$
\begin{aligned}
\left\|(1+|t-| \cdot||)^{-1 / 2}\right\|_{6 / 5, \infty, \varphi} & \leq C\left(\int_{0}^{t+1}(1+|t-r|)^{-3 / 5} r^{3} d r\right)^{5 / 6} \\
& \leq C(1+t)^{5 / 2}\left(\int_{0}^{t+1}(1+|t-r|)^{-3 / 5} d r\right)^{5 / 6} \\
& \leq C(1+t)^{17 / 6} .
\end{aligned}
$$

On the other hand, by Theorem 3.6 (in which we take $p=6$, then $S_{0}=\frac{1}{3}$, $\gamma=\frac{3}{2}$ ) we get

$$
\|u(t, \cdot)\|_{6,2, \chi_{2}} \leq C(1+t)^{-1} \int_{0}^{t}\left[\|F(\tau, \cdot)\|_{3 / 2, \chi_{1}}+(1+\tau)^{-4 / 3}\|F(\tau, \cdot)\|_{1,2, \chi_{2}}\right] d \tau .
$$

Then we obtain

$$
\begin{aligned}
& \left\|(1+|t-| \cdot||)^{-1 / 2} u(t, \cdot)\right\|_{1,2, \chi_{2}} \\
\leq & C(1+t)^{11 / 6} \int_{0}^{t}\left[\|F(\tau, \cdot)\|_{3 / 2, \chi_{1}}+(1+\tau)^{-4 / 3}\|F(\tau, \cdot)\|_{1,2, \chi_{2}}\right] d \tau .
\end{aligned}
$$

Thus, for any given $t \geq 0$, if (3.26) holds, then, in a way similar to the proof of (3.27), it is easy to see that (3.53) follows from (3.54); while, if (3.28) holds, we shall prove that (3.53) still holds.

By Duhamel's principle, we have

$$
u(t, x)=\int_{0}^{t} v(t, x ; \tau) d \tau,
$$

where $v=v(t, x ; \tau)$ is the solution to the following Cauchy problem:

$$
\left\{\begin{array}{l}
\square v=0, \\
t=\tau: v=0, v_{t}=F(\tau, x) .
\end{array}\right.
$$


By (3.22), it is easy to see that for any fixed $\tau \geq 0$,

$$
\operatorname{supp} v \subseteq\{(t, x)|| x \mid \leq t+1\} .
$$

Noting (3.28), when $|x| \geq(t+1) / 2$, we have

$$
t-\tau+|x| \geq \frac{4}{3}(t+1) .
$$

Then, by Lemma 3.1 and noting (3.22), when

$$
t-\tau-|x| \geq 2(\tau+1),
$$

we have

$$
\left|\chi_{2}(t, x) v(t, x ; \tau)\right| \leq C(1+t)^{-3 / 2}(t-\tau-|x|)^{-3 / 2}\|F(\tau, \cdot)\|_{L^{1}\left(\mathbb{R}^{n}\right)} .
$$

Let $\chi(t, x ; \tau)$ be the characteristic function of the set $\{(t, x)|| x \mid \leq t-\tau-$ $2(\tau+1)\}$. Noting $(2.7)$, it follows from (3.59) that

$$
\begin{aligned}
& \left\|(1+|t-| \cdot||)^{-1 / 2} \chi v(t, \cdot ; \tau)\right\|_{1,2, \chi_{2}} \\
\leq & C(1+t)^{-3 / 2} \int_{r \leq t-\tau-2(\tau+1)}(1+|t-r|)^{-1 / 2}(t-\tau-r)^{-3 / 2} r^{3} d r \\
& \quad\|F(\tau, \cdot)\|_{L^{1}\left(\mathbb{R}^{n}\right)} \\
\leq & C(1+t)^{3 / 2} \int_{t-\tau-r \geq 2(\tau+1)}(t-\tau-r)^{-2} d r \cdot\|F(\tau, \cdot)\|_{L^{1}\left(\mathbb{R}^{n}\right)} \\
\leq & C(1+t)^{3 / 2}(1+\tau)^{-1}\|F(\tau, \cdot)\|_{L^{1}\left(\mathbb{R}^{n}\right)} .
\end{aligned}
$$

Using Hölder's inequality and noticing (3.22), we have

$$
\|F(\tau, \cdot)\|_{L^{1}\left(\mathbb{R}^{n}\right)} \leq C\left((1+\tau)^{4 / 3}\|F(\tau, \cdot)\|_{3 / 2, \chi_{1}}+\|F(\tau, \cdot)\|_{1,2, \chi_{2}}\right) .
$$

Hence we get

$$
\begin{aligned}
& \left\|(1+|t-| \cdot||)^{-1 / 2} \chi v(t, \cdot ; \tau)\right\|_{1,2, \chi_{2}} \\
\leq & C(1+t)^{3 / 2}\left((1+\tau)^{1 / 3}\|F(\tau, \cdot)\|_{3 / 2, \chi_{1}}+(1+\tau)^{-1}\|F(\tau, \cdot)\|_{1,2, \chi_{2}}\right) .
\end{aligned}
$$

Let $\varphi$ be the characteristic function of the set $\{(t, x)|| x \mid \leq t+1\}$. Noting (3.57) and using Hölder's inequality, we have

$$
\begin{aligned}
& \left\|(1+|t-| \cdot||)^{-1 / 2}(1-\chi) v(t, \cdot ; \tau)\right\|_{1,2, \chi_{2}} \\
\leq & \left\|(1+|t-| \cdot||)^{-1 / 2} \varphi(1-\chi)\right\|_{6 / 5, \infty}\|v(t, \cdot ; \tau)\|_{6,2, \chi_{2}} .
\end{aligned}
$$


Using (2.7) it is easy to see that

$$
\begin{aligned}
& \left\|(1+|t-| \cdot||)^{-1 / 2} \varphi(1-\chi)\right\|_{6 / 5, \infty} \\
\leq & C(1+t)^{5 / 2}\left(\int_{t-r \leq 3 \tau+2}(1+|t-r|)^{-3 / 5} d r\right)^{5 / 6} \\
\leq & C(1+t)^{5 / 2}(1+\tau)^{1 / 3} .
\end{aligned}
$$

Moreover, by (2.32) (in which we take $n=4, p=6$, then $S_{0}=\frac{1}{3}$ ) and (3.41) we have

$$
\begin{aligned}
\|v(t, \cdot ; \tau)\|_{6,2, \chi_{2}} & \leq C(1+t)^{-1}\|v(t, \cdot ; \tau)\|_{\dot{H}^{1 / 3}\left(\mathbb{R}^{4}\right)} \\
& \leq C(1+t)^{-1}\|F(\tau, \cdot)\|_{\dot{H}^{-2 / 3}\left(\mathbb{R}^{4}\right)}
\end{aligned}
$$

and by (2.47) (in which we take $S_{0}=\frac{2}{3}$, then $q=\frac{3}{2}$ ) we have

(3.66) $\|F(\tau, \cdot)\|_{\dot{H}^{-2 / 3}\left(\mathbb{R}^{4}\right)} \leq C\left(\|F(\tau, \cdot)\|_{3 / 2, \chi_{1}}+(1+\tau)^{-4 / 3}\|F(\tau, \cdot)\|_{1,2, \chi_{2}}\right)$.

Substituting (3.64)-(3.66) into (3.63) gives

$$
\begin{aligned}
& \left\|(1+|t-| \cdot||)^{-1 / 2}(1-\chi) v(t, \cdot ; \tau)\right\|_{1,2, \chi_{2}} \\
\leq & C(1+t)^{3 / 2}\left((1+\tau)^{1 / 3}\|F(\tau, \cdot)\|_{3 / 2, \chi_{1}}+(1+\tau)^{-1}\|F(\tau, \cdot)\|_{1,2, \chi_{2}}\right) .
\end{aligned}
$$

Combining (3.62) and (3.67) yields

$$
\begin{aligned}
& \left\|(1+|t-| \cdot||)^{-1 / 2} v(t, \cdot ; \tau)\right\|_{1,2, \chi_{2}} \\
\leq & C(1+t)^{3 / 2}\left((1+\tau)^{1 / 3}\|F(\tau, \cdot)\|_{3 / 2, \chi_{1}}+(1+\tau)^{-1}\|F(\tau, \cdot)\|_{1,2, \chi_{2}},\right)
\end{aligned}
$$

then (3.53) follows from (3.55).

Theorem 3.11. Under the assumptions of Theorem 3.9, for any integer $N \geq 0$ we have

$$
\begin{aligned}
\sum_{|k| \leq N}\left\|(1+|t-| \cdot||)^{-1 / 2} \Gamma^{k} u(t, \cdot)\right\|_{1,2, \chi_{2}} & \\
\leq C(1+t)^{3 / 2}\left\{\|u(0, \cdot)\|_{\Gamma, N+4,1}+\left\|u_{t}(0, \cdot)\right\|_{\Gamma, N+3,1}\right. & \\
& +\int_{0}^{t}\left[(1+\tau)^{1 / 3}\|F(\tau, \cdot)\|_{\Gamma, N, 3 / 2, \chi_{1}}\right. \\
& \left.\left.+(1+\tau)^{-1}\|F(\tau, \cdot)\|_{\Gamma, N, 1,2, \chi_{2}}\right] d \tau\right\}, \quad \forall t \geq 0,
\end{aligned}
$$

where $C$ is a positive constant. 
Proof. By Lemma 2.1, it suffices to prove

$$
\begin{aligned}
\left\|(1+|t-| \cdot||)^{-1 / 2} u(t, \cdot)\right\|_{1,2, \chi_{2}} \\
\leq C(1+t)^{3 / 2}\left\{\|f\|_{W^{4,1}\left(\mathbb{R}^{n}\right)}+\|g\|_{W^{3,1}\left(\mathbb{R}^{n}\right)}\right. \\
+\int_{0}^{t}\left[(1+\tau)^{1 / 3}\|F(\tau, \cdot)\|_{3 / 2, \chi_{1}}\right. \\
\left.\left.+(1+\tau)^{-1}\|F(\tau, \cdot)\|_{1,2, \chi_{2}}\right] d \tau\right\}, \quad \forall t \geq 0 .
\end{aligned}
$$

Furthermore, by Lemma 3.10 it is only necessary to show that for the solution $u=u(t, x)$ to the Cauchy problem (3.1)-(3.2) in four space dimensions, we have

$$
\begin{aligned}
& \left\|(1+|t-| \cdot||)^{-1 / 2} u(t, \cdot)\right\|_{1,2, \chi_{2}} \\
\leq & C(1+t)^{3 / 2}\left(\|f\|_{W^{4,1}\left(\mathbb{R}^{n}\right)}+\|g\|_{W^{3,1}\left(\mathbb{R}^{n}\right)}\right) .
\end{aligned}
$$

However, (3.71) is a direct consequence of (3.18).

4. Life-span of classical solutions to nonlinear wave equations in four space dimensions.

In this section we shall prove the lower bound estimate (1.8) on the lifespan of classical solutions to Cauchy problem (1.14)-(1.15) for quasilinear wave equations in four space dimensions.

The solution $u$ to Cauchy problem (1.14)-(1.15) can be written as

$$
u=w+u_{\varepsilon},
$$

where $u_{\varepsilon}$ is the solution to the following Cauchy problem for the homogeneous wave equation:

$$
\begin{aligned}
& \square u_{\varepsilon}=0, \\
& t=0: u_{\varepsilon}=\varepsilon \varphi(x),\left(u_{\varepsilon}\right)_{t}=\varepsilon \psi(x) ;
\end{aligned}
$$

while $w$ is the solution to the following Cauchy problem:

$$
\begin{aligned}
& \square w=\sum_{i, j=1}^{4} b_{i j}(u, D u) u_{x_{i} x_{j}}+2 \sum_{j=1}^{4} a_{0 j}(u, D u) u_{t x_{j}}+F_{0}(u, D u), \\
& t=0: w=0, w_{t}=0 .
\end{aligned}
$$


By Sobolev embedding theorem, there exists $E_{0}>0$ so small that

$$
\|f\|_{L^{\infty}\left(\mathbb{R}^{4}\right)} \leq 1, \quad \forall f \in H^{3}\left(\mathbb{R}^{n}\right),\|f\|_{H^{3}\left(\mathbb{R}^{n}\right)} \leq E_{0} .
$$

For any given integer $S \geq 11$ and any given positive numbers $E\left(\leq E_{0}\right)$ and $T$, introduce the following set of functions:

(4.7) $X_{S, E, T}=\left\{\omega(t, x) \mid D_{S, T}(\omega) \leq E, \partial_{t}^{\ell} \omega(0, x)=w_{\ell}^{(0)}(x)(\ell=0,1, \ldots, S+1)\right\}$, where

$$
\begin{aligned}
D_{S, T}(\omega)=\sum_{|i|=1}^{2} \sup _{0 \leq t \leq T}\left\|D^{i} \omega(t, \cdot)\right\|_{\Gamma, S, 2}+\sup _{0 \leq t \leq T}\|\omega(t, \cdot)\|_{\Gamma, S, 2, \chi_{1}} \\
+\sup _{0 \leq t \leq T}(1+t)\|\omega(t, \cdot)\|_{\Gamma, S, 6,2, \chi_{2}} \\
+\sup _{0 \leq t \leq T}(1+t)^{-3 / 2}(\ln (2+t))^{-1}|||\omega(t, \cdot)| \| \\
+\sup _{\substack{0 \leq t \leq T \\
x \in \mathbb{R}^{4}}}(1+t)^{3 / 2}(1+|t-| x||)^{1 / 2} \sum_{|k| \leq S-3}\left|\Gamma^{k} \omega(t, x)\right|,
\end{aligned}
$$

where $\chi_{1}$ is the characteristic function of the set $\{(t, x)|| x \mid \leq(t+1) / 2\}, \chi_{2}=$ $1-\chi_{1}$ and

$$
\|\mid \omega(t, \cdot)\|\left\|=\sum_{|k| \leq S}\right\|(1+|t-| \cdot||)^{-1 / 2} \Gamma^{k} \omega(t, \cdot) \|_{1,2, \chi_{2}} .
$$

Moreover, $w_{0}^{(0)}=w_{1}^{(0)}=0$ and $w_{\ell}^{(0)}(x)(\ell=2, \ldots, S+1)$ are the values of $\partial_{t}^{\ell} w(t, x)$ at $t=0$, formally determined by Cauchy problem (4.4)-(4.5) with (4.1)-(4.3). Obviously, all $w_{\ell}^{(0)}(x)(\ell=0,1, \ldots, S+1)$ are sufficiently smooth functions with compact support.

It is easy to get the following:

Lemma 4.1. Endowed with the metric

$$
\rho(\bar{\omega}, \overline{\bar{\omega}})=D_{S, T}(\bar{\omega}-\overline{\bar{\omega}}), \quad \forall \bar{\omega}, \overline{\bar{\omega}} \in X_{S, E, T}, .
$$

$X_{S, E, T}$ is a nonempty complete metric space, provided that $\varepsilon>0$ is suitably small.

Let $\widetilde{X}_{S, E, T}$ be the subset of $X_{S, E, T}$ composed of all elements $\omega \in X_{S, E, T}$ such that

$$
\operatorname{supp} \omega \subseteq\{(t, x)|| x \mid \leq t+1\} .
$$

The main result in this paper is the following: 
Theorem 4.2. Suppose that $n=4, \alpha=1$ and $\partial_{u}^{2} F(0,0,0) \neq 0$. Under hypotheses (1.16)-(1.22), for any given integer $S \geq 11$, there exist positive constants $\varepsilon_{0}$ and $C_{0}$ with $C_{0} \varepsilon_{0}^{2} \leq E_{0}$ such that for any $\varepsilon \in\left(0, \varepsilon_{0}\right]$, there exists a positive number $T=T(\varepsilon)$ such that Cauchy problem (1.14)-(1.15) admits on $[0, T(\varepsilon)]$ a unique classical solution $u=w+u_{\varepsilon}, u_{\varepsilon}$ being the solution to Cauchy problem (4.2)-(4.3) and $w \in \tilde{X}_{S, C_{0} \varepsilon^{2}, T(\varepsilon)}$, where

$$
T(\varepsilon)=\exp \left\{A \varepsilon^{-2}\right\}-2
$$

and $A$ is a positive constant independent of $\varepsilon$.

Moreover, with eventual modification on a set with zero measure in the variable $t$, we have

$$
\begin{aligned}
& u \in C\left([0, T(\varepsilon)] ; H^{s+1}\left(\mathbb{R}^{4}\right)\right), \\
& u_{t} \in C\left([0, T(\varepsilon)] ; H^{s}\left(\mathbb{R}^{4}\right)\right), \\
& u_{t t} \in C\left([0, T(\varepsilon)] ; H^{s-1}\left(\mathbb{R}^{4}\right)\right) .
\end{aligned}
$$

In order to prove Theorem 4.2, we define a map

$$
M: \omega \rightarrow w=M \omega
$$

by solving the following Cauchy problem for any fixed $\omega \in \tilde{X}_{S, E, T}$ :

$$
\begin{aligned}
& \text { (4.17) } \square w=\hat{F}\left(v, D v, D_{x} D u\right) \\
& \triangleq \sum_{i, j=1}^{4} b_{i j}(v, D v) u_{x_{i} x_{j}}+2 \sum_{j=1}^{4} a_{0 j}(v, D v) u_{t x_{j}}+F_{0}(v, D v), \\
& (4.18) \quad t=0: w=0, w_{t}=0,
\end{aligned}
$$

where

$$
v=\omega+u_{\varepsilon}, \quad u=w+u_{\varepsilon} .
$$

Thus, it is only necessary to prove that there exists $C_{0}>0$ such that the map $M$ possesses a unique fixed point $w \in \widetilde{X}_{S, C_{0} \varepsilon^{2}, T(\varepsilon)}$, provided that $\varepsilon>0$ is suitably small and $T(\varepsilon)$ is given by (4.12).

It is not difficult to get the following two lemmas. 
Lemma 4.3. For any $\omega \in \tilde{X}_{S, E, T}$, with eventual modification on a set with zero measure in $t$, we have

$$
\begin{aligned}
& w=M \omega \in C\left([0, T] ; H^{s+1}\left(\mathbb{R}^{4}\right)\right), \\
& w_{t} \in C\left([0, T] ; H^{s}\left(\mathbb{R}^{4}\right)\right), \\
& w_{t t} \in L^{\infty}\left(0, T ; H^{s-1}\left(\mathbb{R}^{4}\right)\right)
\end{aligned}
$$

and

$$
\operatorname{supp} w \subseteq\{(t, x)|| x \mid \leq t+1\}
$$

Lemma 4.4. For $w=w(t, x)=M \omega, \partial_{t}^{\ell} w(0, x)(\ell=0,1, \ldots, S+2)$ are independent of $\omega \in \tilde{X}_{S, E, T}$, and

$$
\partial_{t}^{\ell} w(0, x)=w_{\ell}^{(0)}(x) \quad(\ell=0,1, \ldots, S+1) .
$$

Furthermore,

$$
\|w(0, \cdot)\|_{\Gamma, S+2, p, q} \leq C \varepsilon^{2},
$$

where $\|w(0, \cdot)\|_{\Gamma, S+2, p, q}$ is the value of $\|w(t, \cdot)\|_{\Gamma, S+2, p, q}$ at $t=0,1 \leq p, q \leq+\infty$ and $C$ is a positive constant independent of $\varepsilon$.

Remark 4.5. For $u=w+u_{\varepsilon}$ we only have

$$
\|u(0, \cdot)\|_{\Gamma, S+2, p, q} \leq C \varepsilon
$$

Comparing with (4.25) suggests the previous iteration with respect to $w$ instead of $u$, which leads to the improved extimate (1.8).

Lemma 4.6. Suppose that $n=4$. For any integer $N \geq 0$, the solution $u_{\varepsilon}(t, x)$ to Cauchy problem (4.2)-(4.3) satisfies

$$
\left\|u_{\varepsilon}(t, \cdot)\right\|_{\Gamma, N, p, q} \leq C(1+t)^{3(1 / p-1 / 2)} \varepsilon, \quad \forall t \geq 0,
$$

where $1 \leq p, q \leq+\infty$ and $C$ is a positive constant.

Proof. Using Lemma 3.3 and noting

$$
\operatorname{supp} u_{\varepsilon} \subseteq\{(t, x)|| x \mid \leq t+1\},
$$

by (2.7)-(2.8) we easily get (4.26). 
Lemma 4.7. Suppose that $n=4$. For any $\omega \in \tilde{X}_{S, E, T}, v=\omega+u_{\varepsilon}$ satisfies

$$
\left\|\left(v, D v, D^{2} v\right)(t, \cdot)\right\|_{\Gamma,[S / 2], \infty} \leq C(1+t)^{-3 / 2}(E+\varepsilon), \quad \forall t \in[0, T],
$$

where $C$ is a positive constant.

Proof. Noting that $S \geq 11$ and by the definition of $X_{S, E, T}$, it is easy to see that

$$
\|\omega(t, \cdot)\|_{\Gamma,[S / 2], \infty} \leq\|\omega(t, \cdot)\|_{\Gamma, S-3, \infty} \leq(1+t)^{-3 / 2} E, \quad \forall t \in[0, T] .
$$

Moreover, by Theorem 2.8 (in which we take $n=4$ and $p=2$ ), similarly we get

$$
\begin{aligned}
\left\|\left(D \omega, D^{2} \omega\right)(t, \cdot)\right\|_{\Gamma,[S / 2], \infty} & \leq C(1+t)^{-3 / 2}\left\|\left(D \omega, D^{2} \omega\right)(t, \cdot)\right\|_{\Gamma, S, 2} \\
& \leq C(1+t)^{-3 / 2} E, \quad \forall t \in[0, T] .
\end{aligned}
$$

Thus, we have

$$
\left\|\left(\omega, D \omega, D^{2} \omega\right)(t, \cdot)\right\|_{\Gamma,[S / 2], \infty} \leq C(1+t)^{-3 / 2} E, \quad \forall t \in[0, T] .
$$

On the other hand, by Lemma 4.6 it is easy to see that

$$
\left\|\left(u_{\varepsilon}, D u_{\varepsilon}, D^{2} u_{\varepsilon}\right)(t, \cdot)\right\|_{\Gamma,[S / 2], \infty} \leq C(1+t)^{-3 / 2} \varepsilon, \quad \forall t \in[0, T] .
$$

The combination of (4.31) and (4.32) yields (4.28).

Lemma 4.8. Suppose that $n=4$. For any function $w(t, x)$ such that (4.23) holds and $D_{S, T}(w)<+\infty$, we have

$$
\begin{aligned}
& \left\|\left(w, D w, D^{2} w\right)(t, \cdot)\right\|_{\Gamma, S-3, p, q} \leq C(1+t)^{-3(1 / 2-1 / p)} D_{S, T}(w), \\
& \quad(2<p \leq+\infty), \\
& \left\|\left(w, D w, D^{2} w\right)(t, \cdot)\right\|_{\Gamma, S-3,2, q} \leq C \sqrt{\ln (2+t)} D_{S, T}(w) .
\end{aligned}
$$

In particular, for any $\omega \in \tilde{X}_{S, E, T}$ we have

$$
\begin{aligned}
& \left\|\left(\omega, D \omega, D^{2} \omega\right)(t, \cdot)\right\|_{\Gamma, S-3, p, q} \leq C(1+t)^{-3(1 / 2-1 / p)} E \\
& \quad(2<p \leq+\infty), \\
& \left\|\left(\omega, D \omega, D^{2} \omega\right)(t, \cdot)\right\|_{\Gamma, S-3,2, q} \leq C \sqrt{\ln (2+t)} E .
\end{aligned}
$$

In (4.33)-(4.36), $t \in[0, T], C$ is a positive constant and $1 \leq q \leq+\infty$. 
Proof. By the definition of $D_{S, T}(w)$ we have

$$
\sum_{|k| \leq S-3}\left|\Gamma^{k} w(t, x)\right| \leq(1+t)^{-3 / 2}(1+|t-| x||)^{-1 / 2} D_{S, T}(w) .
$$

Moreover, by Theorem 2.8 (in which we take $n=4$ and $p=2$ ) and noting the definition of $D_{S, T}(w)$, we have

$$
\begin{aligned}
& \sum_{|k| \leq S-3}\left|\Gamma^{k}\left(D w, D^{2} w\right)(t, x)\right| \\
\leq & C(1+t)^{-3 / 2}(1+|t-| x||)^{-1 / 2}\left\|\left(D w, D^{2} w\right)(t, \cdot)\right\|_{\Gamma, S, 2} \\
\leq & C(1+t)^{-3 / 2}(1+|t-| x||)^{-1 / 2} D_{S, T}(w) .
\end{aligned}
$$

Hence, we get

$$
\sum_{|k| \leq S-3}\left|\Gamma^{k}\left(w, D w, D^{2} w\right)(t, x)\right| \leq C(1+t)^{-3 / 2}(1+|t-| x||)^{-1 / 2} D_{S, T}(w) .
$$

Thus, noting (4.23), when $2<p<+\infty$, we have

$$
\begin{aligned}
\|w(t, \cdot)\|_{\Gamma, S-3, p, q} & \leq C(1+t)^{-3 / 2}\left(\int_{0}^{t+\rho}(1+|t-r|)^{-p / 2} r^{3} d r\right)^{1 / p} D_{S, T}(w) \\
& \leq C(1+t)^{-3(1 / 2-1 / p)}\left(\int_{0}^{t+\rho}(1+|t-r|)^{-p / 2} d r\right)^{1 / p} D_{S, T}(w) \\
& \leq C(1+t)^{-3(1 / 2-1 / p)} D_{S, T}(w)
\end{aligned}
$$

and it is easy to see that the previous estimate is still valid when $p=+\infty$; while, when $p=2$, we have

$$
\begin{aligned}
\|w(t, \cdot)\|_{\Gamma, S-3,2, q} & \leq C(1+t)^{-3 / 2}\left(\int_{0}^{t+\rho}(1+|t-r|)^{-1} r^{3} d r\right)^{1 / 2} D_{S, T}(w) \\
& \leq C\left(\int_{0}^{t+\rho}(1+|t-r|)^{-1} d r\right)^{1 / 2} D_{S, T}(w) \\
& \leq C \sqrt{\ln (2+t)} D_{S, T}(w) .
\end{aligned}
$$

Similarly to Li Ta-tsien and Yu Xin [5], in order to prove Theorem 4.2 it suffices to show the following two lemmas. 
Lemma 4.9. For any $\omega \in \tilde{X}_{S, E, T}, w=M \omega$ satisfies

$$
D_{S, T}(w) \leq C_{1}\left\{\varepsilon^{2}+(R+\sqrt{R})\left(E+D_{S, T}(w)\right)\right\},
$$

where $C_{1}$ is a positive constant and

$$
R=R(\varepsilon, E, T)=E \ln (2+T)+\varepsilon \sqrt{\ln (2+T)} .
$$

Lemma 4.10. Let $\bar{\omega}$, $\overline{\bar{\omega}} \in \tilde{X}_{S, E, T}$. If $\bar{w}=M \bar{\omega}$ and $\overline{\bar{w}}=M \overline{\bar{\omega}}$ also satisfy $\bar{w}, \overline{\bar{w}} \in \tilde{X}_{S, E, T}$, then

$$
D_{S-1, T}(\bar{w}-\overline{\bar{w}}) \leq C_{2}(R+\sqrt{R})\left(D_{S-1, T}(\bar{w}-\overline{\bar{w}})+D_{S-1, T}(\bar{\omega}-\overline{\bar{\omega}})\right),
$$

where $C_{2}$ is a positive constant and $R=R(\varepsilon, E, T)$ is still defined by (4.41).

We shall only prove Theorem 4.9. The proof of Theorem 4.10 is similar.

Proof of Lemma 4.9. We first estimate $\|w(t, \cdot)\|_{\Gamma, S, 2, \chi_{1}}$.

By Theorem 3.5 (in which we take $n=4$, then $q=\frac{4}{3}$ ) and noting Lemma 4.4, we have

$$
\begin{aligned}
\|w(t, \cdot)\|_{\Gamma, S, 2, \chi_{1}} & \\
\leq C(1+t)^{-1} & \left\{\varepsilon^{2}+\int_{0}^{t}\left[(1+\tau)\left\|\hat{F}\left(v, D v, D_{x} D u\right)(\tau, \cdot)\right\|_{\Gamma, S, 4 / 3, \chi_{1}}\right.\right. \\
+ & \left.\left.\left\|\hat{F}\left(v, D v, D_{x} D u\right)(\tau, \cdot)\right\|_{\Gamma, S, 1,2, \chi_{2}}\right] d \tau\right\}, \quad \forall t \in[0, T] .
\end{aligned}
$$

Noting Lemma 4.7, for $E_{0}$ and $\varepsilon_{0}$ suitably small, by Lemma 2.3 and Lemma 2.4 we get

$$
\begin{gathered}
\quad\left\|\hat{F}\left(v, D v, D_{x} D u\right)(\tau, \cdot)\right\|_{\Gamma, S, 4 / 3, \chi_{1}} \\
\leq C\left(\|(v, D v)(\tau, \cdot)\|_{\Gamma,[S / 2], 4, \chi_{1}}\left\|\left(v, D v, D_{x} D u\right)(\tau, \cdot)\right\|_{\Gamma, S, 2, \chi_{1}}\right. \\
\left.\quad+\left\|D_{x} D u(\tau, \cdot)\right\|_{\Gamma,[S / 2], 4, \chi_{1}}\|(v, D v)(\tau, \cdot)\|_{\Gamma, S, 2, \chi_{1}}\right) .
\end{gathered}
$$

Using (2.30) (in which we take $n=4, p=2, q=4$ and $S_{0}=2$ ) and noting that $S \geq 11$, it is easy to see that

$$
\begin{gathered}
\left\|D_{x} D u(\tau, \cdot)\right\|_{\Gamma,[S / 2], 4, \chi_{1}} \leq C(1+\tau)^{-1}\left\|D_{x} D u(\tau, \cdot)\right\|_{\Gamma, S, 2, \chi_{1}}, \\
\|(v, D v)(\tau, \cdot)\|_{\Gamma,[S / 2], 4, \chi_{1}} \leq C(1+\tau)^{-1}\|(v, D v)(\tau, \cdot)\|_{\Gamma, S, 2, \chi_{1}} .
\end{gathered}
$$


Then, noting Lemma 4.6 (in which we take $p=q=2$ ) and the definition of $D_{S, T}(w)$, by (4.17) we have

$$
\begin{aligned}
& \left\|\hat{F}\left(v, D v, D_{x} D u\right)(\tau, \cdot)\right\|_{\Gamma, S, 4 / 3, \chi_{1}} \\
\leq & C(1+\tau)^{-1}\|(v, D v)(\tau, \cdot)\|_{\Gamma, S, 2, \chi_{1}}\left\|\left(v, D v, D_{x} D u\right)(\tau, \cdot)\right\|_{\Gamma, S, 2, \chi_{1}} \\
\leq & C(1+\tau)^{-1}(E+\varepsilon)\left(E+\varepsilon+D_{S, T}(w)\right) \\
\leq & C(1+\tau)^{-1}\left(\varepsilon^{2}+R\left(E+D_{S, T}(w)\right)\right)
\end{aligned}
$$

On the other hand, noting Lemma 4.7 and using Lemma 2.4, for $E_{0}$ and $\varepsilon_{0}$ suitably small, it is easy to get that

$$
\begin{aligned}
& \left\|\hat{F}\left(v, D v, D_{x} D u\right)(\tau, \cdot)\right\|_{\Gamma, S, 1,2, \chi_{2}} \\
& \leq C \sum_{\substack{\left|k_{1}\right|+\left|k_{2}\right| \leq S \\
\left|k_{1}\right| \leq\left|k_{2}\right|}}\left(\left\|\Gamma^{k_{1}}\left(v, D v, D_{x} D u\right)(\tau, \cdot) \Gamma^{k_{2}} v(\tau, \cdot)\right\|_{1,2, \chi_{2}}\right. \\
& +\left\|\Gamma^{k_{1}}\left(v, D v, D_{x} D u\right)(\tau, \cdot) \Gamma^{k_{2}} D v(\tau, \cdot)\right\|_{1,2, \chi_{2}} \\
& \left.+\left\|\Gamma^{k_{1}}(v, D v)(\tau, \cdot) \Gamma^{k_{2}} D_{x} D u(\tau, \cdot)\right\|_{1,2, \chi_{2}}\right) \\
& \triangleq \mathrm{I}+\mathrm{II}+\mathrm{III} \text {. }
\end{aligned}
$$

Noting that $S \geq 11$, by (4.34) and (4.36) (in which we take $q=+\infty$ ) and Lemma 4.6 (in which we take $p=2, q=+\infty$ and $p=q=2$ respectively) we have

$$
\begin{aligned}
\mathrm{II} & \leq C\left\|\left(v, D v, D_{x} D u\right)(\tau, \cdot)\right\|_{\Gamma,[S / 2], 2, \infty}\|D v(\tau, \cdot)\|_{\Gamma, S, 2} \\
& \leq C(E+\varepsilon)\left\{\varepsilon+\sqrt{\ln (2+t)}\left(E+D_{S, T}(w)\right)\right\} \\
& \leq C\left\{\varepsilon^{2}+R\left(E+D_{S, T}(w)\right)\right\} .
\end{aligned}
$$

Similarly, we have

$$
\begin{aligned}
\mathrm{III} & \leq C\|(v, D v)(\tau, \cdot)\|_{\Gamma,[S / 2], 2, \infty}\left\|D_{x} D u(\tau, \cdot)\right\|_{\Gamma, S, 2} \\
& \leq C(\varepsilon+\sqrt{\ln (2+t)} E)\left(\varepsilon+D_{S, T}(w)\right) \\
& \leq C\left\{\varepsilon^{2}+R\left(E+D_{S, T}(w)\right)\right\} .
\end{aligned}
$$

We now estimate I. By (4.19) we have 


$$
\begin{aligned}
\mathrm{I} \leq C \sum_{\substack{\left|k_{1}\right|+\left|k_{2}\right| \leq S \\
\left|k_{1}\right| \leq\left|k_{2}\right|}}\left\{\left\|\Gamma^{k_{1}}\left(u_{\varepsilon}, D u_{\varepsilon}, D_{x} D u_{\varepsilon}\right)(\tau, \cdot) \Gamma^{k_{2}} u_{\varepsilon}(\tau, \cdot)\right\|_{1,2, \chi_{2}}\right. \\
\quad+\left\|\Gamma^{k_{1}}\left(u_{\varepsilon}, D u_{\varepsilon}, D_{x} D u_{\varepsilon}\right)(\tau, \cdot) \Gamma^{k_{2}} \omega(\tau, \cdot)\right\|_{1,2, \chi_{2}} \\
\quad+\left\|\Gamma^{k_{1}}\left(\omega, D \omega, D_{x} D w\right)(\tau, \cdot) \Gamma^{k_{2}} \omega(\tau, \cdot)\right\|_{1,2, \chi_{2}} \\
\left.\quad+\left\|\Gamma^{k_{1}}\left(\omega, D \omega, D_{x} D w\right)(\tau, \cdot) \Gamma^{k_{2}} u_{\varepsilon}(\tau, \cdot)\right\|_{1,2, \chi_{2}}\right\} \\
\triangleq \mathrm{I}_{1}+\mathrm{I}_{2}+\mathrm{I}_{3}+\mathrm{I}_{4} .
\end{aligned}
$$

Noting Lemma 2.4, by Lemma 4.6 (in which we take $p=2, q=+\infty$ and $p=q=2$ respectively) we get

$$
\begin{aligned}
\mathrm{I}_{1} & \leq C\left\|\left(u_{\varepsilon}, D u_{\varepsilon}, D_{x} D u_{\varepsilon}\right)(\tau, \cdot)\right\|_{\Gamma,[S / 2], 2, \infty}\left\|u_{\varepsilon}(\tau, \cdot)\right\|_{\Gamma, S, 2} \\
& \leq C \varepsilon^{2} .
\end{aligned}
$$

Similarly, by Lemma 4.6 (in which we take $p=\frac{6}{5}$ and $q=+\infty$ ) and noting the definition of $D_{S, T}(w)$, we have

$$
\begin{aligned}
\mathrm{I}_{2} & \leq C\left\|\left(u_{\varepsilon}, D u_{\varepsilon}, D_{x} D u_{\varepsilon}\right)(\tau, \cdot)\right\|_{\Gamma,[S / 2], 6 / 5, \infty}\|\omega(\tau, \cdot)\|_{\Gamma, S, 6,2, \chi_{2}} \\
& \leq C \varepsilon E .
\end{aligned}
$$

Moreover, noticing that $S \geq 11$, by the definition of $D_{S, T}(w)$ and $\||\omega|\|$, it is easy to see that

$$
\begin{gathered}
\mathrm{I}_{3} \leq C \sum_{\substack{\left|k_{1}\right|+\left|k_{2}\right| \leq S \\
\left|k_{1}\right| \leq\left|k_{2}\right|}} \|(1+|\tau-| \cdot||)^{1 / 2} \Gamma^{k_{1}}\left(\omega, D \omega, D_{x} D w\right)(\tau, \cdot) \\
\cdot(1+|\tau-| \cdot||)^{-1 / 2} \Gamma^{k_{2}} \omega(\tau, \cdot) \|_{1,2, \chi_{2}} \\
\leq C\left(E+D_{S, T}(w)\right) \ln (2+t) E .
\end{gathered}
$$

Finally, noting that $S \geq 11$, by Lemma 4.6 (in which we take $p=\frac{6}{5}$ and $q=+\infty$ ) and the definition of $D_{S, T}(w)$, we have

$$
\begin{aligned}
\mathrm{I}_{4} & \leq C\left\|\left(\omega, D \omega, D_{x} D w\right)(\tau, \cdot)\right\|_{\Gamma,[S / 2], 6,2, \chi_{2}}\left\|u_{\varepsilon}(\tau, \cdot)\right\|_{\Gamma, S, 6 / 5, \infty} \\
& \leq C \varepsilon\left(E+D_{S, T}(w)\right) .
\end{aligned}
$$


Combining (4.49)-(4.52), it follows from (4.48) that

$$
\begin{aligned}
\mathrm{I} & \leq C\left\{\varepsilon^{2}+(\varepsilon+E \ln (2+t))\left(E+D_{S, T}(w)\right)\right\} \\
& \leq C\left\{\varepsilon^{2}+R\left(E+D_{S, T}(w)\right)\right\} .
\end{aligned}
$$

Substituting (4.53) and (4.46)-(4.47) into (4.45) yields

$$
\left\|\hat{F}\left(v, D v, D_{x} D u\right)(\tau, \cdot)\right\|_{\Gamma, S, 1,2, \chi_{2}} \leq C\left\{\varepsilon^{2}+R\left(E+D_{S, T}(w)\right)\right\} .
$$

Thus, using (4.44) and (4.54), it follows from (4.43) that

$$
\|w(t, \cdot)\|_{\Gamma, S, 2, \chi_{1}} \leq C\left\{\varepsilon^{2}+R\left(E+D_{S, T}(w)\right)\right\} .
$$

We now estimate $\|w(t, \cdot)\|_{\Gamma, S, 6,2, \chi_{2}}$.

By Theorem 3.6 (in which we take $n=4$ and $p=6$, then $S_{0}=\frac{1}{3}, \gamma=\frac{3}{2}$ ) and using Lemma 4.4, we get

$$
\begin{aligned}
& \|w(t, \cdot)\|_{\Gamma, S, 6,2, \chi_{2}} \\
& \leq C(1+t)^{-1}\left\{\varepsilon^{2}+\int_{0}^{t}\left[\left\|\hat{F}\left(v, D v, D_{x} D u\right)(\tau, \cdot)\right\|_{\Gamma, S, 3 / 2, \chi_{1}}\right.\right. \\
& \left.\left.\quad+(1+\tau)^{-4 / 3}\left\|\hat{F}\left(v, D v, D_{x} D u\right)(\tau, \cdot)\right\|_{\Gamma, S, 1,2, \chi_{2}}\right] d \tau\right\} .
\end{aligned}
$$

By Lemma 2.3 and Lemma 2.4, using (2.30) (in which we take $n=4, p=2$, $q=6$ and $\left.S_{0}=2\right)$ and noting Lemma 4.6 and the definition of $D_{S, T}(w)$, we have

$$
\begin{aligned}
& \left\|\hat{F}\left(v, D v, D_{x} D u\right)(\tau, \cdot)\right\|_{\Gamma, S, 3 / 2, \chi_{1}} \\
\leq & C\left(\|(v, D v)(\tau, \cdot)\|_{\Gamma,[S / 2], 6, \chi_{1}}\left\|\left(v, D v, D_{x} D u\right)(\tau, \cdot)\right\|_{\Gamma, S, 2, \chi_{1}}\right. \\
& \left.\quad+\left\|D_{x} D u(\tau, \cdot)\right\|_{\Gamma,[S / 2], 6, \chi_{1}}\|(v, D v)(\tau, \cdot)\|_{\Gamma, S, 2, \chi_{1}}\right) \\
\leq & C(1+\tau)^{-4 / 3}\|(v, D v)(\tau, \cdot)\|_{\Gamma, S, 2, \chi_{1}}\left\|\left(v, D v, D_{x} D u\right)(\tau, \cdot)\right\|_{\Gamma, S, 2, \chi_{1}} \\
\leq & C(1+\tau)^{-4 / 3}(E+\varepsilon)\left(E+\varepsilon+D_{S, T}(w)\right) \\
\leq & C(1+\tau)^{-4 / 3}\left\{\varepsilon^{2}+R\left(E+D_{S, T}(w)\right)\right\} .
\end{aligned}
$$

Putting (4.57) and (4.54) into (4.56) leads to

$$
\|w(t, \cdot)\|_{\Gamma, S, 6,2, \chi_{2}} \leq C(1+t)^{-1}\left\{\varepsilon^{2}+R\left(E+D_{S, T}(w)\right)\right\} .
$$

We next estimate $\||w(t, \cdot) \||$. 
Noting Lemma 4.4 and using (4.57) and (4.54), by Theorem 3.11 we have $(4.59)$

$$
\begin{aligned}
& \|w(t, \cdot)\| \\
\leq & C(1+t)^{3 / 2}\left\{\varepsilon^{2}+\int_{0}^{t}\left[(1+\tau)^{1 / 3}\left\|\hat{F}\left(v, D v, D_{x} D u\right)(\tau, \cdot)\right\|_{\Gamma, S, 3 / 2, \chi_{1}}\right.\right. \\
& \left.\left.+(1+\tau)^{-1}\left\|\hat{F}\left(v, D v, D_{x} D u\right)(\tau, \cdot)\right\|_{\Gamma, S, 1,2, \chi_{2}}\right]\right\} d \tau \\
\leq & C(1+t)^{3 / 2}\left\{\varepsilon^{2}+\ln (2+t)\left[\varepsilon^{2}+R\left(E+D_{S, T}(w)\right)\right]\right\} \\
\leq & C(1+t)^{3 / 2} \ln (2+t)\left\{\varepsilon^{2}+R\left(E+D_{S, T}(w)\right)\right\} .
\end{aligned}
$$

Now we estimate

$$
\sum_{|k| \leq S-3}\left|\Gamma^{k} w(t, x)\right| .
$$

By Theorem 3.9 and noting Lemma 4.4, we have

$$
\begin{aligned}
& \sum_{|k| \leq S-3}\left|\Gamma^{k} w(t, x)\right| \\
\leq & C(1+t)^{-3 / 2}(1+|t-| x||)^{-1 / 2}\left(\varepsilon^{2}+\sup _{0 \leq \tau \leq t}\left\|\hat{F}\left(v, D v, D_{x} D u\right)(\tau, \cdot)\right\|_{\Gamma, S, 1}\right) .
\end{aligned}
$$

Using Sobolev embedding theorem on the unit sphere $S^{n-1}$, it is easy to see that

$$
\begin{aligned}
& \left\|\hat{F}\left(v, D v, D_{x} D u\right)(\tau, \cdot)\right\|_{\Gamma, S, 1} \\
= & \left\|\hat{F}\left(v, D v, D_{x} D u\right)(\tau, \cdot)\right\|_{\Gamma, S, 1, \chi_{1}}+\left\|\hat{F}\left(v, D v, D_{x} D u\right)(\tau, \cdot)\right\|_{\Gamma, S, 1, \chi_{2}} \\
\leq & C\left(\left\|\hat{F}\left(v, D v, D_{x} D u\right)(\tau, \cdot)\right\|_{\Gamma, S, 1, \chi_{1}}+\left\|\hat{F}\left(v, D v, D_{x} D u\right)(\tau, \cdot)\right\|_{\Gamma, S, 1,2, \chi_{2}}\right) .
\end{aligned}
$$

Using Lemma 2.3 and Lemma 2.4, and noting Lemma 4.6 and the definition of $D_{S, T}(w)$, we easily get

$$
\begin{aligned}
& \left\|\hat{F}\left(v, D v, D_{x} D u\right)(\tau, \cdot)\right\|_{\Gamma, S, 1, \chi_{1}} \\
\leq & C\|(v, D v)(\tau, \cdot)\|_{\Gamma, S, 2, \chi_{1}}\left\|\left(v, D v, D_{x} D u\right)(\tau, \cdot)\right\|_{\Gamma, S, 2, \chi_{1}} \\
\leq & C(E+\varepsilon)\left(E+\varepsilon+D_{S, T}(w)\right) \\
\leq & C\left\{\varepsilon^{2}+R\left(E+D_{S, T}(w)\right)\right\} .
\end{aligned}
$$

Then, using (4.54), by (4.60) we get

$$
\begin{aligned}
& \sum_{|k| \leq S-3}\left|\Gamma^{k} w(t, x)\right| \\
\leq & C(1+t)^{-3 / 2}(1+|t-| x||)^{-1 / 2}\left\{\varepsilon^{2}+R\left(E+D_{S, T}(w)\right)\right\} .
\end{aligned}
$$


Finally, we estimate

$$
\sum_{|i|=1}^{2} \sup _{0 \leq t \leq T}\left\|D^{i} w(t, \cdot)\right\|_{\Gamma, S, 2} .
$$

For any multi-index $k(|k| \leq S)$, by applying $\Gamma^{k}$ and $\Gamma^{k} D$ to both sides of (4.17), respectively, we can get the following energy integral formula:

$$
\begin{aligned}
& \left\|D \Gamma^{k} w(t, \cdot)\right\|_{L^{2}\left(\mathbb{R}^{4}\right)}^{2}+\left\|\left(\Gamma^{k} D w(t, \cdot)\right)_{t}\right\|_{L^{2}\left(\mathbb{R}^{4}\right)}^{2} \\
& +\sum_{i, j=1}^{4} \int_{\mathbb{R}^{4}} a_{i j}(v, D v)(t, x)\left(\Gamma^{k} D w(t, x)\right)_{x_{i}}\left(\Gamma^{k} D w(t, x)\right)_{x_{j}} d x \\
& =\left\|D \Gamma^{k} w(0, \cdot)\right\|_{L^{2}\left(\mathbb{R}^{4}\right)}^{2}+\left\|\left(\Gamma^{k} D w(0, \cdot)\right)_{t}\right\|_{L^{2}\left(\mathbb{R}^{4}\right)}^{2} \\
& +\sum_{i, j=1}^{4} \int_{\mathbb{R}^{4}} a_{i j}(v, D v)(0, x)\left(\Gamma^{k} D w(0, x)\right)_{x_{i}}\left(\Gamma^{k} D w(0, x)\right)_{x_{j}} d x \\
& +\sum_{i, j=1}^{4} \int_{0}^{t} \int_{\mathbb{R}^{4}} \frac{\partial a_{i j}(v, D v)(\tau, x)}{\partial \tau}\left(\Gamma^{k} D w(\tau, x)\right)_{x_{i}}\left(\Gamma^{k} D w(\tau, x)\right)_{x_{j}} d x d \tau \\
& -2 \sum_{i, j=1}^{4} \int_{0}^{t} \int_{\mathbb{R}^{4}} \frac{\partial a_{i j}(v, D v)(\tau, x)}{\partial x_{i}}\left(\Gamma^{k} D w(\tau, x)\right)_{x_{j}}\left(\Gamma^{k} D w(\tau, x)\right)_{\tau} d x d \tau \\
& -2 \sum_{j=1}^{4} \int_{0}^{t} \int_{\mathbb{R}^{4}} \frac{\partial a_{0 j}(v, D v)(\tau, x)}{\partial x_{j}}\left(\Gamma^{k} D w(\tau, x)\right)_{\tau}\left(\Gamma^{k} D w(\tau, x)\right)_{\tau} d x d \tau \\
& +2 \int_{0}^{t} \int_{\mathbb{R}^{4}} G_{k}(\tau, x)\left(\Gamma^{k} D w(\tau, x)\right)_{\tau} d x d \tau \\
& +2 \int_{0}^{t} \int_{\mathbb{R}^{4}} g_{k}(\tau, x)\left(\Gamma^{k} D w(\tau, x)\right)_{\tau} d x d \tau \\
& =\left\|D \Gamma^{k} w(0, \cdot)\right\|_{L^{2}\left(\mathbb{R}^{4}\right)}^{2}+\left\|\left(\Gamma^{k} D w(0, \cdot)\right)_{t}\right\|_{L^{2}\left(\mathbb{R}^{4}\right)}^{2} \\
& +\sum_{i, j=1}^{4} \int_{\mathbb{R}^{4}} a_{i j}(v, D v)(0, x)\left(\Gamma^{k} D w(0, x)\right)_{x_{i}}\left(\Gamma^{k} D w(0, x)\right)_{x_{j}} d x \\
& +\mathrm{I}+\mathrm{II}+\mathrm{III}+\mathrm{IV}+\mathrm{V},
\end{aligned}
$$


in which

$$
\begin{aligned}
G_{k}=\sum_{i, j=1}^{4}\{ & \left(\Gamma^{k} D\left(b_{i j}(v, D v) w_{x_{i} x_{j}}\right)-b_{i j}(v, D v) \Gamma^{k} D w_{x_{i} x_{j}}\right) \\
& \left.+b_{i j}(v, D v)\left(\Gamma^{k} D w_{x_{i} x_{j}}-\left(\Gamma^{k} D w\right)_{x_{i} x_{j}}\right)\right\} \\
& +2 \sum_{j=1}^{4}\left\{\left(\Gamma^{k} D\left(a_{0 j}(v, D v) w_{t x_{j}}\right)-a_{0 j}(v, D v) \Gamma^{k} D w_{t x_{j}}\right)\right. \\
& \left.+a_{0 j}(v, D v)\left(\Gamma^{k} D w_{t x_{j}}-\left(\Gamma^{k} D w\right)_{t x_{j}}\right)\right\} \\
g_{k}=\Gamma^{k} D( & F_{0}(v, D v)+\sum_{i, j=1}^{4} b_{i j}(v, D v)\left(u_{\varepsilon}\right)_{x_{i} x_{j}} \\
& \left.+2 \sum_{j=1}^{4} a_{0 j}(v, D v)\left(u_{\varepsilon}\right)_{t x_{j}}\right) \\
& +\sum_{|i| \leq|k|} C_{k i} \Gamma^{i} \hat{F}\left(v, D v, D_{x} D w\right),
\end{aligned}
$$

where $\hat{F}\left(v, D v, D_{x} D w\right)$ is defined by (4.17) and $C_{k i}$ are constants.

Using Lemma 2.4 and noting Lemma 2.2 , it is easy to see that

$$
\text { I, II, III } \leq C(E+\varepsilon) D_{S, T}^{2}(w) .
$$

We now estimate $\left\|G_{k}(\tau, \cdot)\right\|_{L^{2}\left(\mathbb{R}^{4}\right)}$.

Using Lemma 4.6 and Lemma 4.8 and noting that $S \geq 11$, by Lemma 2.3 and Lemma 2.4 we get

$$
\begin{aligned}
& \left\|\left(\Gamma^{k} D\left(b_{i j}(v, D v) w_{x_{i} x_{j}}\right)-b_{i j}(v, D v) \Gamma^{k} D w_{x_{i} x_{j}}\right)(\tau, \cdot)\right\|_{L^{2}\left(\mathbb{R}^{4}\right)} \\
\leq & C\left\{\left\|D b_{i j}(v, D v)(\tau, \cdot)\right\|_{\Gamma, S, 2}\left\|w_{x_{i} x_{j}}(\tau, \cdot)\right\|_{\Gamma,[S / 2]+1, \infty}\right. \\
& \left.\quad+\left\|b_{i j}(v, D v)(\tau, \cdot)\right\|_{\Gamma,[S / 2]+1, \infty}\left\|w_{x_{i} x_{j}}(\tau, \cdot)\right\|_{\Gamma, S, 2}\right\} \\
\leq & C(1+\tau)^{-3 / 2}(E+\varepsilon) D_{S, T}(w) .
\end{aligned}
$$

Moreover, by Lemma 2.1 and Lemma 4.7 and noting the definition of $D_{S, T}(w)$, it is easy to see that

$$
\begin{aligned}
& \left\|b_{i j}(v, D v)\left(\Gamma^{k} D w_{x_{i} x_{j}}-\left(\Gamma^{k} D w\right)_{x_{i} x_{j}}\right)(\tau, \cdot)\right\|_{L^{2}\left(\mathbb{R}^{4}\right)} \\
\leq & C\left\|b_{i j}(v, D v)(\tau, \cdot)\right\|_{L^{\infty}\left(\mathbb{R}^{4}\right)}\left\|D_{x}^{2} w(\tau, \cdot)\right\|_{\Gamma, S, 2} \\
\leq & C(1+\tau)^{-3 / 2}(E+\varepsilon) D_{S, T}(w) .
\end{aligned}
$$


For the terms consisting of $a_{0 j}$ in $G_{k}$, similar estimates hold. Therefore,

$$
\left\|G_{k}(\tau, \cdot)\right\|_{L^{2}\left(\mathbb{R}^{4}\right)} \leq C(1+\tau)^{-3 / 2}(E+\varepsilon) D_{S, T}(w),
$$

then

$$
\mathrm{IV} \leq C(E+\varepsilon) D_{S, T}^{2}(w) .
$$

We next estimate $\left\|g_{k}(\tau, \cdot)\right\|_{L^{2}\left(\mathbb{R}^{4}\right)}$.

By Lemma 2.3 and Lemma 2.4, we have

$$
\begin{aligned}
& \left\|\Gamma^{k} D F_{0}(v, D v)(\tau, \cdot)\right\|_{L^{2}\left(\mathbb{R}^{4}\right)} \\
\leq \| D F_{0} & (v, D v)(\tau, \cdot) \|_{\Gamma, S, 2} \\
\leq C\{\| & (v, D v)(\tau, \cdot)\left\|_{\Gamma,[S / 2], \infty}\right\|\left(D v, D^{2} v\right)(\tau, \cdot) \|_{\Gamma, S, 2} \\
& +\left\|\left(D v, D^{2} v\right)(\tau, \cdot)\right\|_{\Gamma,[S / 2], \infty}\|D v(\tau, \cdot)\|_{\Gamma, S, 2} \\
& +\left\|\left(D v, D^{2} v\right)(\tau, \cdot)\right\|_{\Gamma,[S / 2], \infty, \chi_{1}}\|v(\tau, \cdot)\|_{\Gamma, S, 2, \chi_{1}} \\
& \left.+\left\|\left(D v, D^{2} v\right)(\tau, \cdot)\right\|_{\Gamma,[S / 2], 3, \infty, \chi_{2}}\|v(\tau, \cdot)\|_{\Gamma, S, 6,2, \chi_{2}}\right\} \\
\leq C\{\| & (v, D v)(\tau, \cdot)\left\|_{\Gamma,[S / 2], \infty}\right\|\left(D v, D^{2} v\right)(\tau, \cdot) \|_{\Gamma, S, 2} \\
& +\left\|\left(D v, D^{2} v\right)(\tau, \cdot)\right\|_{\Gamma,[S / 2], \infty}\left(\|D v(\tau, \cdot)\|_{\Gamma, S, 2}+\|v(\tau, \cdot)\|_{\Gamma, S, 2, \chi_{1}}\right) \\
& \left.+\left\|\left(D v, D^{2} v\right)(\tau, \cdot)\right\|_{\Gamma,[S / 2], 3, \infty}\|v(\tau, \cdot)\|_{\Gamma, S, 6,2, \chi_{2}}\right\} \\
\triangleq J_{1}+ & J_{2}+J_{3} .
\end{aligned}
$$

Noting Lemma 4.6 and the Definition of $D_{S, T}(w)$, by Lemma 4.7 it is easy to see that

$$
J_{1}, J_{2} \leq C(1+t)^{-3 / 2}(E+\varepsilon)^{2} .
$$

On the other hand, by Lemma 4.6 we have

$$
\begin{aligned}
\left\|\left(D u_{\varepsilon}, D^{2} u_{\varepsilon}\right)(\tau, \cdot)\right\|_{\Gamma,[S / 2], 3, \infty} & \leq C(1+t)^{-1 / 2} \varepsilon, \\
\left\|u_{\varepsilon}(\tau, \cdot)\right\|_{\Gamma, S, 6,2} & \leq C(1+t)^{-1} \varepsilon .
\end{aligned}
$$

Moreover, noting that $S \geq 11$ and using Lemma 4.8, we have

$$
\left\|\left(D \omega, D^{2} \omega\right)(\tau, \cdot)\right\|_{\Gamma,[S / 2], 3, \infty} \leq C(1+t)^{-1 / 2} E .
$$

Then, noting (4.19) and the definition of $D_{S, T}(\omega)$, we get

$$
J_{3} \leq C(1+t)^{-3 / 2}(E+\varepsilon)^{2} .
$$


Thus, we have

$$
\left\|\Gamma^{k} D F_{0}(v, D v)(\tau, \cdot)\right\|_{L^{2}\left(\mathbb{R}^{4}\right)} \leq C(1+t)^{-3 / 2}(E+\varepsilon)^{2} .
$$

Furthermore,

$$
\begin{aligned}
& \left\|\Gamma^{k} D\left(\sum_{i, j=1}^{4} b_{i j}(v, D v)\left(u_{\varepsilon}\right)_{x_{i} x_{j}}\right)(\tau, \cdot)\right\|_{L^{2}\left(\mathbb{R}^{4}\right)} \\
& \leq \sum_{i, j=1}^{4}\left\|\Gamma^{k}\left(D b_{i j}(v, D v) \cdot\left(u_{\varepsilon}\right)_{x_{i} x_{j}}\right)(\tau, \cdot)\right\|_{L^{2}\left(\mathbb{R}^{4}\right)} \\
& \quad+\sum_{i, j=1}^{4}\left\|\Gamma^{k}\left(b_{i j}(v, D v)\left(D u_{\varepsilon}\right)_{x_{i} x_{j}}\right)(\tau, \cdot)\right\|_{L^{2}\left(\mathbb{R}^{4}\right)} \\
& \triangleq K_{1}+K_{2} .
\end{aligned}
$$

Noting Lemma 4.6, Lemma 4.7 and the definition of $X_{S, E, T}$, by Lemma 2.3 and Lemma 2.4 we have

$$
\begin{aligned}
& K_{1} \leq\left\|D b_{i j}(v, D v)(\tau, \cdot)\right\|_{\Gamma,[S / 2], \infty}\left\|\left(u_{\varepsilon}\right)_{x_{i} x_{j}}(\tau, \cdot)\right\|_{\Gamma, S, 2} \\
&+\left\|D b_{i j}(v, D v)(\tau, \cdot)\right\|_{\Gamma, S, 2}\left\|\left(u_{\varepsilon}\right)_{x_{i} x_{j}}(\tau, \cdot)\right\|_{\Gamma,[S / 2], \infty} \\
& \leq C\left(\left\|\left(D v, D^{2} v\right)(\tau, \cdot)\right\|_{\Gamma,[S / 2], \infty}\left\|\left(u_{\varepsilon}\right)_{x_{i} x_{j}}(\tau, \cdot)\right\|_{\Gamma, S, 2}\right. \\
&\left.\quad+\left\|\left(D v, D^{2} v\right)(\tau, \cdot)\right\|_{\Gamma, S, 2}\left\|\left(u_{\varepsilon}\right)_{x_{i} x_{j}}(\tau, \cdot)\right\|_{\Gamma,[S / 2], \infty}\right) \\
& \leq C \varepsilon(1+t)^{-3 / 2}(E+\varepsilon) .
\end{aligned}
$$

Moreover, similarly to (4.68)-(4.69), by Lemma 2.3 and Lemma 2.4 we get

$$
\begin{aligned}
K_{2} \leq C\left\{\|(v, D v)(\tau, \cdot)\|_{\Gamma,[S / 2], \infty}\left\|\left(D u_{\varepsilon}\right)_{x_{i} x_{j}}(\tau, \cdot)\right\|_{\Gamma, S, 2}\right. \\
+\left(\|D v(\tau, \cdot)\|_{\Gamma, S, 2}+\|v(\tau, \cdot)\|_{\Gamma, S, 2, \chi_{1}}\right)\left\|\left(D u_{\varepsilon}\right)_{x_{i} x_{j}}(\tau, \cdot)\right\|_{\Gamma,[S / 2], \infty} \\
\left.\quad+\|v(\tau, \cdot)\|_{\Gamma, S, 6,2, \chi_{2}}\left\|\left(D u_{\varepsilon}\right)_{x_{i} x_{j}}(\tau, \cdot)\right\|_{\Gamma,[S / 2], 3, \infty}\right\} \\
\leq C \varepsilon(1+t)^{-3 / 2}(E+\varepsilon) .
\end{aligned}
$$

For the corresponding terms consisting of $a_{0 j}$ in $g_{k}$, similar estimates hold. Hence, we get

$$
\begin{aligned}
& \quad\left\|\Gamma^{k} D\left(\sum_{i, j=1}^{4} b_{i j}(v, D v)\left(u_{\varepsilon}\right)_{x_{i} x_{j}}+2 \sum_{j=1}^{4} a_{0 j}(v, D v)\left(u_{\varepsilon}\right)_{t x_{j}}\right)(\tau, \cdot)\right\|_{L^{2}\left(\mathbb{R}^{4}\right)} \\
& \leq C \varepsilon(1+t)^{-3 / 2}(E+\varepsilon) .
\end{aligned}
$$


Moreover, similarly to (4.70), by Lemma 4.8 we easily get

$$
\begin{aligned}
& \left\|\left(\sum_{i, j=1}^{4} b_{i j}(v, D v) w_{x_{i} x_{j}}+2 \sum_{j=1}^{4} a_{0 j}(v, D v) w_{t x_{j}}\right)(\tau, \cdot)\right\|_{\Gamma, S, 2} \\
\leq & C(1+t)^{-3 / 2}(E+\varepsilon) D_{S, T}(w) .
\end{aligned}
$$

Similarly, we have

$$
\begin{aligned}
& \left\|F_{0}(v, D v)(\tau, \cdot)\right\|_{\Gamma, S, 2} \\
\leq & C\left\{\|(v, D v)(\tau, \cdot)\|_{\Gamma,[S / 2], \infty}\left(\|D v(\tau, \cdot)\|_{\Gamma, S, 2}+\|v(\tau, \cdot)\|_{\Gamma, S, 2, \chi_{1}}\right)\right. \\
& \left.\quad+\|(v, D v)(\tau, \cdot)\|_{\Gamma,[S / 2], 3, \infty}\|v(\tau, \cdot)\|_{\Gamma, S, 6,2, \chi_{2}}\right\} \\
& \quad C(1+t)^{-3 / 2}(E+\varepsilon)^{2} .
\end{aligned}
$$

The combination of (4.69)-(4.72) yields

$$
\left\|g_{k}(\tau, \cdot)\right\|_{L^{2}\left(\mathbb{R}^{4}\right)} \leq C(1+t)^{-3 / 2}(E+\varepsilon)\left(E+\varepsilon+D_{S, T}(w)\right),
$$

then

$$
\mathrm{V} \leq C(E+\varepsilon)\left(E+\varepsilon+D_{S, T}(w)\right) D_{S, T}(w) .
$$

Using (4.65), (4.67) and (4.74) and noting Lemma 2.2, Lemma 4.4 and (1.21), it follows from (4.62) that

$$
\sum_{|i|=1}^{2}\left\|D^{i} w(t, \cdot)\right\|_{\Gamma, S, 2}^{2} \leq C\left\{\varepsilon^{4}+(E+\varepsilon)\left(E+\varepsilon+D_{S, T}(w)\right) D_{S, T}(w)\right\} .
$$

Noting that

$$
(E+\varepsilon)^{2} D_{S, T}(w) \leq C\left(E^{2}+\varepsilon^{2}\right) D_{S, T}(w)
$$

and

$$
E^{2} D_{S, T}(w) \leq C\left(E^{3}+E D_{S, T}^{2}(w)\right) \leq C E\left(E+D_{S, T}(w)\right)^{2},
$$

it comes from (4.75) that

$$
\sum_{|i|=1}^{2}\left\|D^{i} w(t, \cdot)\right\|_{\Gamma, S, 2}^{2} \leq C\left\{\varepsilon^{4}+(E+\varepsilon)\left(E+D_{S, T}(w)\right)^{2}+\varepsilon^{2} D_{S, T}(w)\right\},
$$

then, by Young's inequality we get

$$
\begin{aligned}
\sum_{|i|=1}^{2}\left\|D^{i} w(t, \cdot)\right\|_{\Gamma, S, 2} & \leq C\left\{\varepsilon^{2}+\sqrt{E+\varepsilon}\left(E+D_{S, T}(w)\right)\right\}+\frac{1}{2} D_{S, T}(w) \\
& \leq C\left\{\varepsilon^{2}+\sqrt{R}\left(E+D_{S, T}(w)\right)\right\}+\frac{1}{2} D_{S, T}(w) .
\end{aligned}
$$

The combination of (4.55), (4.58)-(4.59), (4.61) and (4.76) leads to the desired estimate (4.40). The proof of Lemma 4.9 is complete. 
Let $C_{0}=3 \max \left(C_{1}, C_{2}\right)$, where $C_{1}$ and $C_{2}$ are the constants given in Lemma 4.9 and Lemma 4.10 respectively. By Li Ta-tsien and Yu Xin [5], for any $\varepsilon \in\left(0, \varepsilon_{0}\right]$, where $\varepsilon_{0}>0$ is a small number such that $C_{0} \varepsilon_{0}^{2} \leq E_{0}$, if there exists $T=T(\varepsilon)$ such that

$$
R(\varepsilon, E(\varepsilon), T(\varepsilon))+\sqrt{R(\varepsilon, E(\varepsilon), T(\varepsilon))} \leq \frac{1}{C_{0}},
$$

where

$$
E(\varepsilon)=C_{0} \varepsilon^{2},
$$

then the map $M$ has a unique fixed point $w \in \tilde{X}_{S, E(\varepsilon), T(\varepsilon)}$.

Noting (4.41), (4.77) holds if $T(\varepsilon)$ is given by (4.12). The desired conclusion (1.8) follows directly from (4.12).

\section{REFERENCES}

[1] D. Christodoulou, Global solutions of nonlinear hyperbolic equations for small initial data, Comm. Pure Appl. Math. 39 (1986), 267-282.

[2] Li TA-TSIEN \& CHEN YUN-MEI, Initial value problems for nonlinear wave equations, Comm. Partial Differential Equations 13 (1988), 383-422.

[3] L. HÖRmANDER, On the fully non-linear Cauchy problem with small data II, Microlocal Analysis and Nonlinear Waves (M. Beals, R. Melrose and J. Rauch, eds.), Vol. 30, IMA Volumes in Mathematics and its Applications, Springer-Verlag, Berlin, 1991, pp. 51-81.

[4] H. LindBlad, On the lifespan of solutions of nonlinear wave equations with small initial data, Comm. Pure Appl. Math. 43 (1990), 445-472.

[5] Li TA-TSIEN 8 YU XIN, Life-span of classical solutions to fully nonlinear wave equations, Comm. Partial Differential Equations 16 (1991), 909-940.

[6] Li TA-TSIEN \& ZHOU YI, Life-span of classical solutions to fully nonlinear wave equations II, Nonlinear Anal. 19 (1992), 833-853.

[7] Li TA-Tsien, YU Xin \& ZHOU YI, Durée de vie des solutions régulières pour les équations des ondes non linéaires unidimensionnelles, C. R. Acad. Sci. Paris Sér. I Math. 312 (1991), 103-105.

[8] L Life-span of classical solutions to one dimensional nonlinear wave equations, Chinese Ann. Math. Ser. B 13 (1992), 266-279.

[9] _ Problème de Cauchy pour les équations des ondes non linéaires avec petites données initiales, C. R. Acad. Sci. Paris Sér. I Math. 312 (1991), 337-340.

[10] LI TA-TSIEN \& ZHOU YI, Life-span of classical solutions to nonlinear wave equations in two space dimensions, J. Math. Pures Appl. 73 (1994), 223-249.

[11] Life-span of classcial solutions to nonlinear wave equations in two space dimensions II, J. Partial Differential Equations 6 (1993), 17-38.

[12] Nonlinear stability for two space dimensional wave equations with higher order perturbation, Nonlinear World 1 (1994), 35-58.

[13] Li TA-TSIEn \& CHEN Yun-MEI, Global Classical Solutions for Nonlinear Evolution Equations, Pitman Monographs and Surveys in Pure and Applied Mathematics 45, Longman Scientific \& Technical, 1992. 
[14] LI TA-TSIEN, Life-span of classical solutions to nonlinear wave equations, Qualitative Aspects and Applications of Nonlinear Evolution Equations (T. T. Li and P. De Mottoni, eds.), World Scientific, Singapore, 1991, p. 43-76.

[15] H. LindBlAD, Blow up for solutions of $\square u=|u|^{p}$ with small initial data, Thesis, Lund, 1989.

[16] ZHOU YI, Life-span of classical solutions to $u_{t t}-u_{x x}=|u|^{1+\alpha}$, Chinese Ann. Math. Ser. B 13 (1992), 230-243.

[17] Life span of classical solutions to $\square u=|u|^{p}$ in two space dimensions, Chinese Ann. Math. Ser. B 14 (1993), 225-236.

[18] Blow up of classical solutions to $\square u=|u|^{p}$ in three space dimensions, J. Partial Differential Equations 5 (1992), 21-32.

[19] F. JoHN, Blow-up of solutions of nonlinear wave equations in three space dimensions, Manuscripta Math. 28 (1979), 235-268.

[20] R. T. GLASSEY, finite-time blow-up for solutions of nonlinear wave equations, Math. Z. 177 (1981), 323-340.

[21] Existence in the large for $\square u=F(u)$ in two space dimensions, Math. Z. 178 (1981), 223-261.

[22] T. C. SIDERIS, Nonexistence of global solutions to semilinear wave equations in high dimensions, J. Differential Equations 52 (1984), 378-406.

[23] J. SchaEffer, The equation $u_{t t}-\Delta u=|u|^{p}$ for the critical value of $p$, Proc. Royal Soc. Edinburgh 101A (1985), 31-44.

[24] T. C. Sideris, Private communication, 1993.

[25] S. Klainerman, Uniform decay estimates and the Lorentz invariance of the classical wave equations, Comm. Pure Appl. Math. 38 (1985), 321-332.

[26] - Remarks on the global Sobolev inequality in the Minkowski space $R^{n+1}$, Comm. Pure Appl. Math. 40 (1987), 111-116.

[27] Global existence for nonlinear wave equations, Comm. Pure Appl. Math. 33 (1980), 43-101.

[28] L. Hörmander, $L^{1}, L^{\infty}$ estimates for the wave operator, Analyse Mathématique et Applications, Gauthier-Villars, Paris, 1988, pp. 211-234.

LI TA-TSIEN

Department of Mathematics

Fudan University

Shanghai 200433 China

\section{ZHOU YI}

Institute of Mathematics

Fudan University

Shanghai 200433 China

Received: November 1st, 1994; revised: February 15th, 1995. 